\title{
Analyse de la rugosité obtenue par un nouveau procédé de tribofinition
}

\author{
Maxence Bigerelle ${ }^{1,2}$, Alain Iost $^{1, a}$ et Philippe Rocher ${ }^{3}$ \\ 1 Laboratoire de Métallurgie Physique et Génie des Matériaux, CNRS UMR 8517, ENSAM, 8 boulevard Louis XIV, \\ 59046 Lille Cedex, France \\ 2 Laboratoire Roberval, FRE 2833, UTC/CNRS, Centre de Recherches de Royallieu, BP 20529, 60205 Compiègne, France \\ 3 G.R.B., UPRES EA 1049, Faculté de Médecine, 59045 Lille Cedex, France
}

Reçu le 27 octobre 2004 ; accepté le 8 mars 2007

\begin{abstract}
Résumé - Nous proposons dans cet article d'analyser un nouveau procédé de polissage automatique : Ecoclean, qui est développé principalement pour la préparation de prothèses dentaires. En faisant varier les conditions expérimentales (matériau étudié, durée de traitement, présence ou non d'acide, présence ou non d'aiguilles) nous obtenons des états de surface différents. Pour chaque échantillon l'état de surface est caractérisé à l'aide d'un rugosimètre tactile et le calcul d'une centaine de paramètres de rugosité. Nous recherchons ensuite à l'aide du logiciel MESRUG, que nous avons développé dans notre laboratoire, les paramètres de rugosité qui sont les plus pertinents pour caractériser l'état de surface en fonction des conditions expérimentales. Le polissage automatique est comparé au polissage manuel. Cette méthode peut être utilisée pour définir les conditions expérimentales permettant d'obtenir un état de surface répondant à des critères de rugosité déterminés.
\end{abstract}

Mots clés : Alliages dentaires / rugosité / statistiques bootstrap / tribologie / érosion / abrasion

Abstract - Analysis of the roughness obtained by a new polishing process. Usually, corrosion and mechanical resistance of dental prosthesis are affected by the surface roughness of the implant materials. This study aimed at evaluating the ability of a new polishing process: Ecoclean. For different dental-alloys, polishing was performed by varying testing conditions. A computer-based bootstrap method developed in our laboratory is then used to calculate more than one hundred roughness parameters from results recording on these samples by tactile profilometer and to correlate the roughness parameters with experimental conditions. The results were compared with mechanical polished samples one. This method allows us to determine the appropriate experimental conditions to obtain a given set of roughness parameters.

Key words: Dental alloys / roughness / bootstrap statistics / tribology / erosion / abrasion

\section{Introduction}

La nature morphologique de la surface d'un matériau peut conditionner certaines réactions mécaniques (usure), chimiques (corrosion) ou biochimiques (adhésion cellulaire) [1]. Cette surface peut être caractérisée topographiquement par la rugosité qui est la mesure de l'altitude d'un profil. Nous proposons dans cet article d'étudier l'état de surface d'alliages dentaires polis à l'aide d'une méthode mécano-chimique originale, le procédé de polissage (ECOCLEAN ${ }^{\mathrm{TM}}$ ) développé par la société UGIN'DENTAIRE.

Dans un premier temps, l'efficacité du polissage en fonction de sa durée est étudiée sur 6 alliages dentaires, préalablement grenaillés. Dans un deuxième temps, l'efficacité du milieu corrosif est étudiée par deux campagnes

a Auteur correspondant : alain.iost@lille.ensam.fr d'essais afin de dissocier l'effet mécanique de l'effet chimique : pour quantifier l'usure mécanique, le milieu acide est remplacé par de l'eau; pour quantifier l'érosion chimique les aiguilles sont enlevées du système rotatif et remplacées par des agitateurs magnétiques. Finalement on compare le procédé utilisé avec le polissage manuel traditionnel des échantillons.

\section{Matériaux, méthodes expérimentales et essais}

\subsection{Matériaux}

Nous avons utilisé six alliages dentaires $(10 \times 10 \times$ $1 \mathrm{~mm})$ commercialisés par la société Matalor. Les compositions chimiques (Tab. 1) et les caractéristiques 


\section{Nomenclature}

\begin{tabular}{|ll|}
\hline$A_{1}$ & Aire de pics \\
$A_{2}$ & Aire des vallées \\
$A H D(c)$ & Taux de portance à l'altitude $c$ du profil \\
$A_{\mathrm{r}}$ & Distance pics à pics des motifs (norme Cnomo) \\
CTE-PC & Nombre de pics par pouce \\
$C(\tau)$ & Fonction d'autocorrélation \\
$f$ & Fréquence \\
$G$ & Nombre de points d'inflexion du profil \\
$G(f)$ & Densité spectrale de puissance \\
$L_{0}$ & Longueur développée $L_{0}$ \\
$m_{0}$ & Moment d'ordre zéro du spectre \\
$M_{\mathrm{r} 1}$ & \% de pics (norme DIN) \\
$M_{\mathrm{r} 2}$ & \% de vallées (norme DIN) \\
$R_{\mathrm{a}}$ & Rugosité arithmétique \\
$R_{\mathrm{i}}$ & Amplitude des pics dans un motif (norme Cnomo) \\
$R_{\mathrm{k}}$ & Amplitude moyenne de la rugosité (norme DIN) \\
$R_{\mathrm{pk}}$ & Amplitude du régime des pics (norme DIN) \\
$R_{\mathrm{pm}}$ & Amplitude moyenne des pics (norme ISO) \\
$R_{\mathrm{q}}$ & Rugosité quadratique \\
$R_{\mathrm{vk}}$ & Amplitude du régime des vallées (norme DIN) \\
$S_{\mathrm{k}}$ & Mesure de la dissymétrie (skewness) \\
$S_{\mathrm{m}}$ & Largeur des pics (norme DIN) \\
$\Delta$ & Dimension fractale calculée par la méthode AMN \\
$\Delta_{\mathrm{a}}$ & Pente moyenne des profils \\
$\delta$ & $R_{\mathrm{k}}>$ Ordonnée $=$ Pente $>R_{\mathrm{pk}}>R_{\mathrm{vk}}=A_{2}=A_{1}>M_{\mathrm{r} 2}>M \mathrm{r} 1$ \\
& Couche limite d'un fluide \\
\hline
\end{tabular}

mécaniques (Tab. 2) sont conformes aux normes Iso 1562, Din 13906, Niom et Prothesor. Les échantillons sont d'abord polis au papier abrasif 1200, puis grenaillés pendant $10 \mathrm{~s}$ à partir d'une distance de tir de $2,5 \mathrm{~cm}$ sous une pression de 3 bars à l'aide de billes de carbure de silicium de diamètre $110 \mu \mathrm{m}$.

\subsection{Procédé de polissage}

\subsubsection{Polissage traditionnel}

Le polissage est effectué sur des pièces coulées à l'aide d'instruments mécaniques et de produits de polissage. La vitesse de rotation des outils pour le surfaçage et le polissage final se situe entre 5000 et 15000 tours par minute. Les outils sont adaptés au degré de polissage souhaité. Des disques caoutchouc ou des polissoirs souples sont utilisés pour le surfaçage, puis des brossettes pour le polissage plus fin. Le brillantage se fait soit à l'aide de brossettes à polir, soit à l'aide de polissoirs.

\subsubsection{Polissage par le procédé Ecoclean}

Le procédé (Ecoclean ${ }^{\mathrm{TM}}$ ) utilise le principe de polissage suivant :

1) Un échantillon de faibles dimensions est plongé dans un bain à base d'acide chlorhydrique.

2) Des aiguilles en acier inoxydable, baignant dans ce bain, sont animées d'un mouvement rotatif (1500 tours.min ${ }^{-1}$ ) grâce à un champ magnétique.
3) Au contact de la surface, les aiguilles provoquent, dans un premier temps, un décapage.

4) Si le mouvement est entretenu plus de $15 \mathrm{~min}$, les aiguilles polissent la surface par érosion (enlèvement de matière).

Les avantages présumés de cette polisseuse sont les suivants :

1) Gain de temps : la seule manipulation consiste à plonger les échantillons dans le bain d'acide et à programmer le temps de polissage.

2) Homogénéité : les aiguilles balayent la surface aléatoirement et produisent une surface uniforme. Comme le polissage mécanique conventionnel d'échantillons de faible taille est délicat, l'amélioration de l'homogénéité due à ce procédé est d'autant plus marquée que les dimensions de l'échantillon sont faibles.

3) Accessibilité aux surfaces de géométries complexes (bridges).

4) Élimination de l'oxydation dans les intrados les plus inaccessibles.

\subsection{Campagnes d'essais}

Trois campagnes d'essais sont effectuées sur les six échantillons d'alliages précieux qui sont d'abord polis au papier abrasif 1200 puis grenaillés. Les résultats sont comparés à ceux obtenus sur les échantillons polis manuellement. 
Tableau 1. Composition chimique des alliages dentaires étudiés en pourcentage massique.

\begin{tabular}{|c|c|c|c|c|c|c|c|c|c|}
\hline A & Corad & $\begin{array}{c}\text { Co (\%) } \\
61\end{array}$ & $\begin{array}{c}\text { Cr (\%) } \\
26\end{array}$ & $\begin{array}{c}\text { Mo (\%) } \\
10\end{array}$ & $\begin{array}{c}\text { Si (\%) } \\
2\end{array}$ & & & & \\
\hline & & $\mathrm{Au}(\%)$ & $\mathrm{Pt}(\%)$ & $\mathrm{Pd}(\%)$ & $\operatorname{Ag}(\%)$ & $\mathrm{Cu}(\%)$ & $\mathrm{Zn}(\%)$ & $\operatorname{In}(\%)$ & $\mathrm{Ga}(\%)$ \\
\hline B & Cerapall 2 & 2 & 0 & 79 & 0 & 6,9 & 0 & 4,5 & 5,5 \\
\hline $\mathrm{C}$ & V-Gnathos Plus & 85,9 & 11,7 & 0 & 0 & 0 & 1,5 & $<1$ & 0 \\
\hline $\mathrm{D}$ & V-92 & 78,8 & 9,9 & 7,8 & 0 & 0 & 0 & 3,5 & 0 \\
\hline $\mathrm{E}$ & Belor 3 & 71 & 2 & 2 & 9 & 14,5 & $<2$ & 0 & 0 \\
\hline $\mathrm{F}$ & V-Delta SF & 51,5 & 0 & 38,5 & 0 & 0 & 0 & 8,5 & $<2$ \\
\hline
\end{tabular}

Tableau 2. Caractéristiques mécaniques des alliages dentaires étudiés.

\begin{tabular}{|c|c|c|c|c|c|c|}
\hline & & $\begin{array}{c}\text { Dureté } \\
\text { HV }\end{array}$ & $\begin{array}{l}\text { Densité } \\
\text { g.cm }\end{array}$ & $\begin{array}{c}\text { Limite élastique } 0,2 \\
\%\end{array}$ & $\begin{array}{c}\text { Allongement } \\
\%\end{array}$ & $\begin{array}{c}\text { Module d'élasticité } \\
\text { MPa }\end{array}$ \\
\hline$\overline{\mathrm{A}}$ & Corad & 380 & 8,3 & 600 & 4 & \\
\hline B & Cerapall 2 & 280 & 11,4 & 580 & 35 & 126500 \\
\hline $\mathrm{C}$ & V-Gnathos Plus & 190 & 18,9 & 560 & 6 & 94000 \\
\hline $\mathrm{D}$ & V-92 & 190 & 17,65 & 450 & 10 & 96500 \\
\hline $\mathrm{E}$ & Belor 3 & 160 & 15,10 & 350 & 30 & 80000 \\
\hline $\mathrm{F}$ & V-Delta SF & 210 & 14,45 & 480 & 25 & 119000 \\
\hline
\end{tabular}

\subsubsection{Polissage Ecoclean (campagne 1)}

Les échantillons sont polis par le procédé Ecoclean avec des temps d'immersion de 5, 10, 15, 20 ou 30 min, 1 et $2 \mathrm{~h}$.

\subsubsection{Deuxième campagne}

Le milieu acide est remplacé par de l'eau dans le but de quantifier l'effet mécanique du système de polissage. Les temps d'immersion sont de 5, 10, 15, 20, 30 min, 1 et $2 \mathrm{~h}$. Le protocole d'utilisation de la machine est resté similaire à celui utilisé lors des deux premières campagnes.

\subsubsection{Troisième campagne}

Pour quantifier l'effet corrosif du système de polissage et ne pas endommager mécaniquement la surface, nous avons remplacé les aiguilles par des agitateurs métalliques aimantés dont la surface est recouverte d'un caoutchouc afin de reproduire les mouvements turbulents du fluide et ne pas changer la cinétique de la réaction de corrosion. Les temps d'immersion sont de $30 \mathrm{~min}, 1,2$ et $4 \mathrm{~h}$.

\subsection{Mesure de la rugosité}

La rugosité est mesurée sur les échantillons initiaux avant polissage (grenaillés), sur les échantillons polis manuellement selon le protocole décrit dans la section précédente. Les mesures sont effectuées à l'aide d'un rugosimètre tactile bidimensionnel (Perthen) sans filtrage. La longueur de palpage est de $1 \mathrm{~mm}$ et le nombre de points échantillonnés est de 8000. 10 mesures de rugosité sont effectuées sur chaque échantillon et chaque essai est doublé (total : $5 \times 6 \times 2 \times 10=600$ profils). La figure 1 présente différents profils de rugosité obtenus pour l'échantillon Cerapall 2.

Remarque : Il est important de noter qu'à chaque essai correspond un échantillon différent qui est sablé puis introduit dans la machine de polissage. Cette méthodologie offre les avantages suivants :

1) Les conclusions concernant l'influence des caractéristiques mécaniques sur l'aptitude au polissage ne risquent pas de dépendre d'une hétérogénéité locale de la structure du matériau.

2) Les mesures des états de surface ne sont pas corrélées entre elles. Il est donc possible de tester la répétitivité et la fiabilité du polissage. En effet, pour une campagne donnée, lorsque nous étudions deux échantillons polis à des temps différents, la rugosité du premier échantillon n'est pas corrélée avec la mesure du second. Certes, le bruit de mesure serait très nettement minimisé si les mesures étaient effectuées successivement sur le même échantillon à des temps de polissage différents. Mais l'objectif de cette étude est de tester la qualité de polissage de ce procédé et non l'analyse de l'évolution de la rugosité d'un échantillon.

3) Les conclusions concernant l'évolution de la rugosité au cours du processus d'érosion ne risquent pas d'être biaisées par l'état sablé de l'échantillon puisque, à chaque analyse de la topographie d'un échantillon, correspond un sablage différent.

4) Les conclusions liées à la mesure de la rugosité d'un échantillon ne sont pas influencées par la mesure précédente. En effet, 10 mesures de rugosité sont faites sur chaque échantillon. La mesure par profilométrie tactile entraîne la création d'un sillon de taille non négligeable (interaction palpeur-matériau). Il est donc impératif d'éviter de mesurer cette déformation lors d'une mesure ultérieure. Les dimensions des échantillons n'excèdent pas $1 \mathrm{~cm}^{2}$ et il est délicat de ne pas avoir recouvrement 

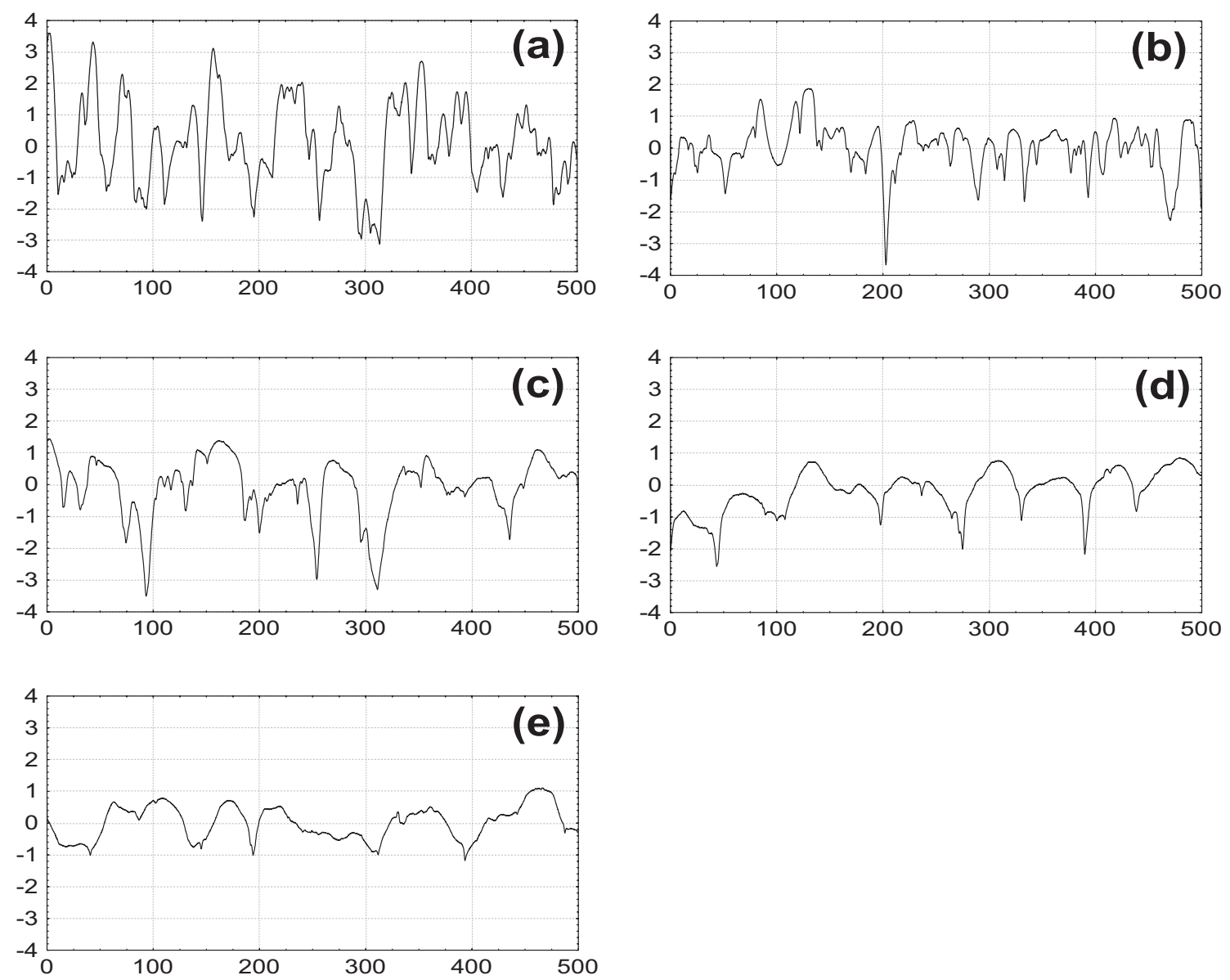

Fig. 1. Évolution des profils de rugosité pour l'échantillon Cerapall 2 au cours du processus de polissage. Les profils sont mesurés après des durées de polissage de $0^{\prime}(\mathrm{a}), 5^{\prime}$ (b), $15^{\prime}$ (c), 30 (d) et $60^{\prime}$ (e). Les hauteurs et longueurs d'évaluation des profils sont indiquées en $\mu \mathrm{m}$.

avec le profil mesuré. Cependant, il est possible avec certaines précautions expérimentales, d'éviter cet artefact. Ces précautions ne seraient plus applicables si les mesures devenaient trop nombreuses. De plus, la surface altérée par la mesure de rugosité risque de modifier le régime hydrodynamique et par conséquent le processus d'érosion de la surface.

\section{Résultats et discussion}

Pour chaque échantillon et chaque profil de rugosité nous calculons 101 paramètres de rugosité qui sont classés en 5 catégories :

1) Paramètres d'amplitude $\left(R_{\mathrm{a}}, R_{\mathrm{t}}, S_{\mathrm{k}}, E_{\mathrm{k}} \ldots\right)$,

2) Paramètres spectraux (longueur d'autocorrélation, moments spectraux...),

3) Paramètres fractals (dimension fractale),

3) Paramètres d'Abbott $\left(R_{\mathrm{k}}, R_{\mathrm{vk}}, R_{\mathrm{pk}} \ldots\right)$,

4) Paramètres CNOMO $\left(A_{\mathrm{r}}, R, W \ldots\right)$,

5) Paramètres « de pics $»($ nombre de pics, Sm, ...).

Nous utilisons ensuite notre logiciel Mesrug [2] qui permet de classer à l'aide d'une méthode originale Bootstrap les paramètres qui optimisent au mieux une équation d'analyse de la variance [3]. Le principe consiste à effectuer un sur-échantillonnage des données avec répétitions qui permet le calcul d'un estimateur avec ses incertitudes associées. Dans notre cas, la méthode statistique retenue est l'analyse de variance. Cette dernière fournit une statistique connue sous le nom du $F$ de Fisher. Plus cette valeur est importante, plus discriminant est l'estimateur du paramètre de rugosité vis-à-vis de la classe considérée. Le Bootstrap permet donc de donner un intervalle de confiance à cette valeur. Le classement par ordre décroissant des valeurs des $F$, obtenu pour chaque paramètre de rugosité, permet d'ordonner les paramètres de rugosité en fonction de leur pertinence, dans ce cas décroissante.

\subsection{Campagne 1}

Dans un premier temps nous allons rechercher les paramètres de rugosité qui discriminent la topographie de surface en fonction du temps de polissage (Fig. 2). Pour chacune des deux séries, les paramètres de rugosité sont classés par ordre croissant de discrimination. Les mesures de rugosité sont regroupées dans une même classe et nous 


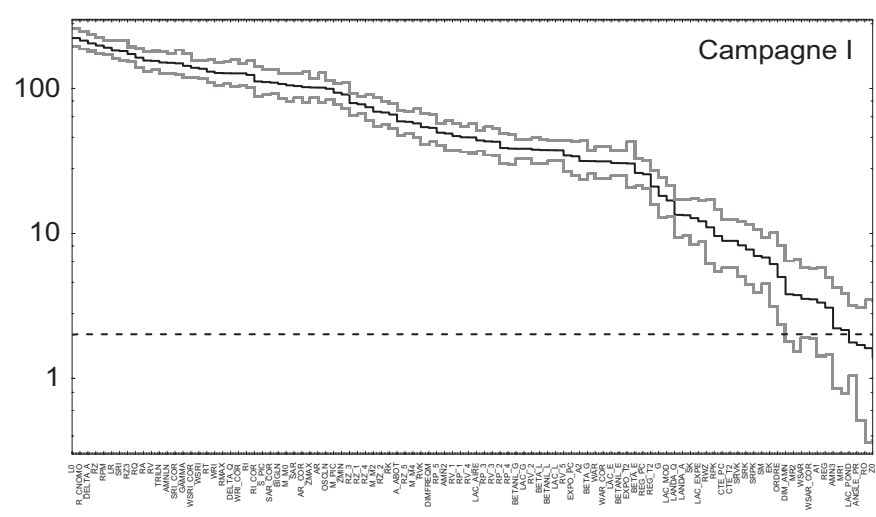

Fig. 2. Classement des paramètres de rugosité de la campagne 1 par l'analyse de la variance bootstrapée. Les paramètres sont classés par $\boldsymbol{F}$ décroissant (du plus influent vers le moins influent). Les limites des intervalles de confiance à $95 \%$ sont représentées en lignes discontinues. La ligne horizontale représente la valeur critique du $\boldsymbol{F}$ en dessous de laquelle le paramètre de rugosité n'est plus significatif.

recherchons par la méthode du Bootstrap quels sont les paramètres qui minimisent la variance intra-classe tout en maximisant la variance inter-classes. Ce rapport $\boldsymbol{F}$ des deux variances pondérées par le nombre de degrés de liberté est calculé pour les 101 paramètres retenus pour l'étude. Ce rapport est d'autant plus élevé que le paramètre discrimine au mieux les différents temps de polissage et donc l'effet du polissage Ecoclean en surface. Cette méthodologie d'analyse ne suppose aucune relation fonctionnelle entre les temps de polissage et le paramètre étudié. Cette étape est une étape intermédiaire qui permet de classer les paramètres discriminants. En effet, si une relation fonctionnelle existe entre la durée de polissage et un paramètre de rugosité, alors la différence entre les mesures des paramètres doit être élevée. Cependant, la topographie de surface est aléatoire et toute mesure de rugosité est entachée d'une variabilité importante. Cette variabilité entraîne une variation de la mesure de $\boldsymbol{F}$ qui peut être calculée par la méthode Bootstrap. La figure 2 présente l'évolution de $\boldsymbol{F}$ (trait plein) et de son intervalle de confiance à $95 \%$ (pointillés). La ligne horizontale représente le seuil critique $(5 \%)$ en dessous duquel il n'est plus possible d'affirmer que $\boldsymbol{F}>1$ et donc que le paramètre considéré ne discrimine plus les effets du polissage. Par un raisonnement similaire, si cette ligne est incluse dans l'intervalle de confiance, il n'est pas possible d'affirmer, ni d'infirmer, l'aspect discriminatoire de la surface. De ce fait, par une visualisation rapide de ce diagramme, il est possible :

1) D'analyser l'influence générale du procédé de polissage sur la rugosité de la surface : plus le $\boldsymbol{F}$ moyen sera élevé et plus le procédé de polissage modifiera les surfaces.

2) De classer les paramètres discriminants du plus pertinent vers le moins pertinent (de la gauche vers la droite).

3) De déterminer les paramètres invariants qui caractérisent la surface vis-à-vis du procédé de polissage. En effet, si la limite inférieure de l'intervalle de confiance est en dessous de la ligne horizontale, la valeur de ce paramètre est indépendante du temps de polissage.

4) De regrouper les paramètres « équivalents » vis-àvis d'un procédé de polissage. Tous les paramètres qui possèdent une intersection commune seront considérés comme équivalents vis-à-vis du caractère discriminatoire, ce qui ne signifie pas que deux paramètres ayant le même indice de discrimination $(\boldsymbol{F})$ sont corrélés. En effet, deux paramètres corrélés théoriquement peuvent très bien se voir attribuer par notre méthode un caractère discriminant très différent vis-à-vis du polissage. Par exemple, en supposant la répartition des amplitudes gaussienne $\varphi(h)$ alors :

$$
\varphi(h)=\frac{1}{R_{\mathrm{q}} \sqrt{2 \pi}} \exp \left(-\frac{h^{2}}{2 R_{\mathrm{q}}}\right)
$$

Le $R_{\mathrm{a}}$ sera donné par :

$$
R_{\mathrm{a}}=\int_{-\infty}^{+\infty}|h| \varphi(h) \mathrm{d} h=2 \int_{0}^{+\infty} h \varphi(h) \mathrm{d} h=R_{\mathrm{q}} \sqrt{\frac{2}{\pi}} \approx 0,8 R_{\mathrm{q}}
$$

De même, si $m_{0}$ est le moment d'ordre 0 de la fonction de densité spectrale $G(f)$, alors $m_{0}=\int_{0}^{+\infty} G(f) \mathrm{d} f$ qui est la valeur prise par la fonction d'auto-corrélation $C(\tau)$ pour $\tau=0$ (théorème de Wiener-Kitchine). Par définition $C(0)=R_{\mathrm{q}}^{2}$ et alors $R_{\mathrm{a}}=0,8 \sqrt{m_{0}}$. Nous avons, pour tous les échantillons sablés et polis par le procédé Ecoclean, tracé l'évolution du $R_{\mathrm{a}}$ en fonction du $R_{\mathrm{q}}$ (Fig. 3a) et en fonction de la racine du moment spectral d'ordre zéro (Fig. 3b). Sur ces graphiques, la ligne d'équation $R_{\mathrm{a}}=$ $0,8 R_{\mathrm{q}}$ est représentée. Nous constatons les faits suivants :

1 ) La corrélation théorique $R_{\mathrm{a}}=0,8 R_{\mathrm{q}}$ est assez bien respectée quand le $R_{\mathrm{q}}$ est calculé par une sommation.

2) La corrélation $R_{\mathrm{a}}=0.8 R_{\mathrm{q}}$ est minimisée si le $R_{\mathrm{q}}$ est calculé par la théorie spectrale $\left(R_{\mathrm{a}} \approx 0,6 \sqrt{m_{0}}\right)$ ce qui s'explique par un artefact de la transformée de Fourier. En effet, quand le $R_{\mathrm{q}}$ croît, souvent il y a déplacement des raies du spectre vers les basses fréquences et le problème du choix de la fenêtre de mesure devient prépondérant.

3) Le bruit sur la détermination du $R_{\mathrm{q}}$ est plus faible que le bruit sur la détermination de $\sqrt{m_{0}}$ malgré une utilisation moyenne du spectre.

Cet exemple montre qu'il existe des corrélations mathématiques, sous des hypothèses parfois délicates à vérifier, entre les paramètres. Cependant la détermination numérique de ceux-ci peut être biaisée ou peu précise, à cause des phénomènes d'échantillonnage et de choix de la fenêtre. Il est alors nécessaire d'analyser la pertinence des paramètres sans a priori sur leur éventuelle corrélation.

D'après la figure 2 nous constatons que les paramètres les plus pertinents pour représenter l'évolution de la rugosité en fonction du temps de polissage sont : $L_{0}, R_{\mathrm{pm}}$, $A_{\mathrm{r}}$, Delta $_{\mathrm{a}}, R_{\mathrm{i}}$ et $S_{\mathrm{ri}}$. Nous omettons les paramètres de ce groupe qui sont auto-corrélés de part leur définition mathématique. Par exemple, le paramètre $R_{\text {cnomo }}$ est corrélé avec le paramètre $R_{\mathrm{i}}, L_{0}$ avec $L R$. La figure 4 montre la corrélation entre la développée du profil $\left(L_{0}\right)$ et la moyenne arithmétique de la pente des profils (Delta $\left.a_{\mathrm{a}}\right)$. 

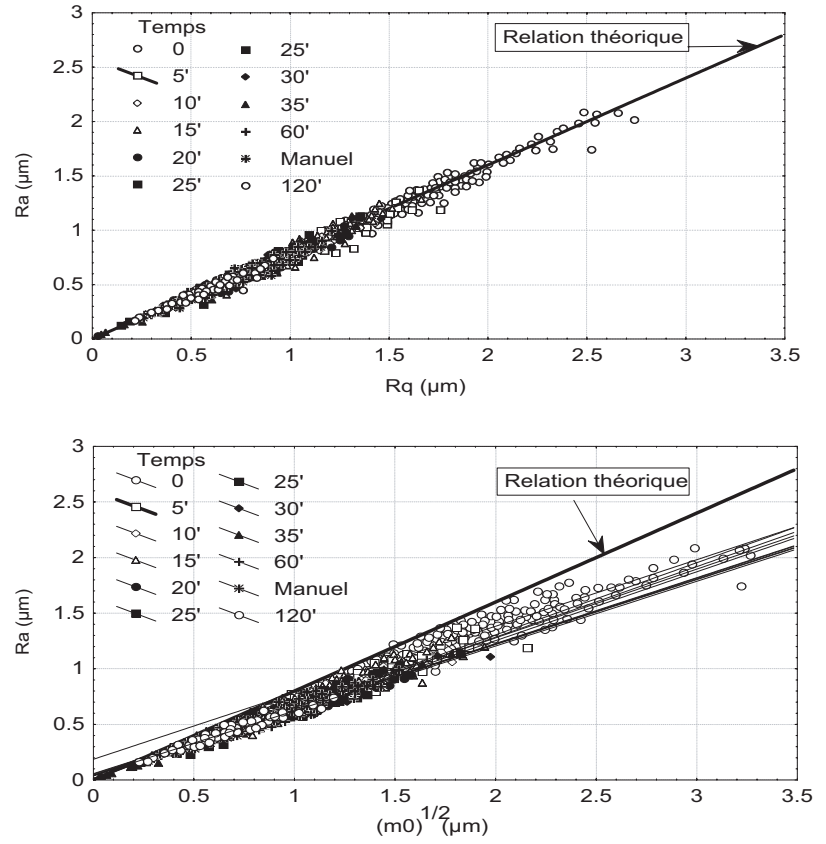

\begin{tabular}{|c|c|c|c|c|c|}
\hline$t$ & $R_{\mathrm{q}}$ & $\sqrt{m_{0}}$ & $t$ & $R_{\mathrm{q}}$ & $\sqrt{m_{0}}$ \\
\hline $0^{\prime}$ & 0,792 & 0,698 & $25^{\prime}$ & 0,779 & 0,642 \\
\hline $5^{\prime}$ & 0,779 & 0,680 & $30^{\prime}$ & 0,786 & 0,643 \\
\hline $10^{\prime}$ & 0,774 & 0,656 & $35^{\prime}$ & 0,781 & 0,634 \\
\hline $15^{\prime}$ & 0,778 & 0,656 & $60^{\prime}$ & 0,794 & 0,644 \\
\hline $20^{\prime}$ & 0,773 & 0,643 & $120^{\prime}$ & 0,786 & 0,640 \\
\hline & & & Manuel & 0,768 & 0,653 \\
\hline
\end{tabular}

Fig. 3. Analyse de la corrélation entre $R_{\mathrm{q}}$ et $R_{\mathrm{a}}$ (a), puis de la corrélation entre $\sqrt{m_{0}}$ et $R_{\mathrm{a}}$ (b) pour les échantillons de la campagne 1 pris à différents temps de polissage (de 0 à $2 \mathrm{~h}$ ) et pour l'échantillon poli manuellement. La droite en trait plein représente la relation théorique entre $R_{\mathrm{a}}$ et $R_{\mathrm{q}}$ sous l'hypothèse des amplitudes gaussiennes $\left(R_{\mathrm{a}}=0,8 R_{\mathrm{q}}\right)$. Par régression (sans intercept), nous obtenons les relations $R_{\mathrm{a}}=\alpha R_{\mathrm{q}}$. Le coefficient $\alpha$ est donné dans le tableau ci-dessus.

L'analyse de ce graphique montre que la développée, comme la moyenne des pentes du profil, diminue avec le temps de polissage. La corrélation, calculée par régression sur l'ensemble des points, sans distinguer le temps de polissage, suit une loi puissance $\Delta_{\mathrm{a}}=0,041\left(L_{0}-500\right)^{0,56}$. On montre statistiquement que cette loi puissance est indépendante du temps de polissage et que considérer les deux paramètres pour l'analyse du procédé de polissage n'apporte aucune information supplémentaire. Nous retiendrons le paramètre $L_{0}$ qui possède la plus faible dispersion sur le diagramme de discrimination. En reproduisant ce type d'analyse pour les paramètres, nous retenons les suivants :

1) La longueur développée $L_{0}$,

2) La dimension fractale calculée par la méthode des auto-corrélations moyennées normées [4],

3) L'amplitude moyenne des pics (norme ISO) $: R_{\mathrm{pm}}$,

4) La largeur des pics (norme DIN) : $S_{\mathrm{m}}$,

5) Le nombre de pics par pouces : CTE-PC,

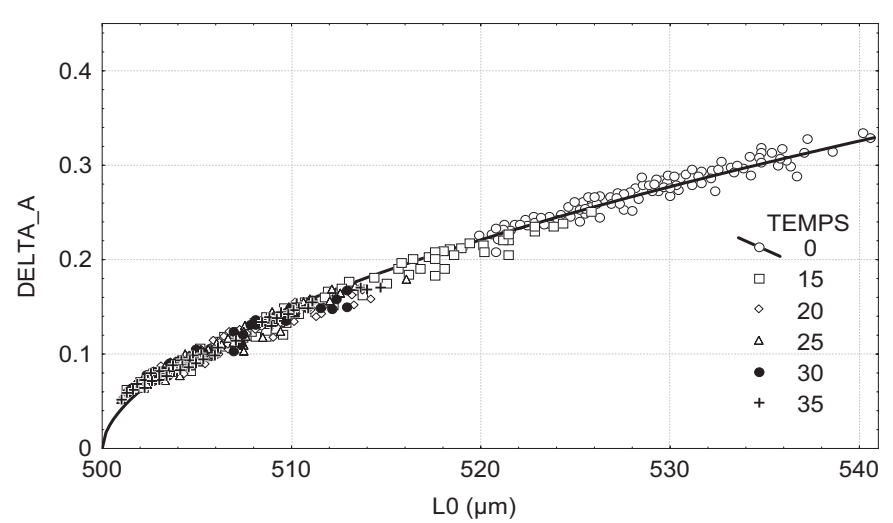

Fig. 4. Analyse de la corrélation entre les paramètres de rugosité $\Delta_{\mathrm{a}}$ et $L_{0}$, pour les essais de la campagne 1 et différents temps de polissage $\left(0^{\prime}, 15^{\prime}, 20^{\prime}, 25^{\prime}, 30^{\prime}\right.$ et $\left.35^{\prime}\right)$. La courbe est la fonction puissance ajustant au mieux (au sens des moindres carrés) les points expérimentaux.

6) La distance pics à pics des motifs (norme CNOMO, modifiée par nos soins pour être applicable à cette longueur particulière de palpage) : $A_{\mathrm{r}}$,

7) L'amplitude des pics dans un motif (norme CNOMO modifiée) : $R_{\mathrm{i}}$,

8) L'amplitude du régime des pics (norme DIN) : $R_{\mathrm{pk}}$,

9) L'amplitude du régime des vallées (norme DIN) : $R_{\mathrm{vk}}$,

10) La rugosité arithmétique : $R_{\mathrm{a}}$. Bien que ce paramètre soit moins discriminant et présente une corrélation marquée avec le $R_{\mathrm{pm}}$, il est conservé car il est traditionnellement le plus utilisé pour caractériser un état de surface.

L'évolution des paramètres de rugosité choisis est représentée en fonction du temps de polissage (Fig. 5). Les paramètres d'amplitude chutent $\left(R_{\mathrm{a}}, R_{\mathrm{pm}}, R_{\mathrm{i}}\right.$ et $\left.R_{\mathrm{pk}}\right)$ fortement pendant les 15 premières $\min$ de polissage. L'échantillon Corad (A), qui possède la dureté la plus élevée $(H V=380)$, réagit différemment des autres échantillons vis-à-vis d'une grande catégorie de critères $\left(R_{\mathrm{vk}}, R_{\mathrm{i}}, A r, S_{\mathrm{m}}, \operatorname{Pics}, L_{0}\right)$ à l'exception des paramètres $\left(R_{\mathrm{pm}}\right.$ et $\left.R_{\mathrm{pk}}\right)$.

La longueur développée décroît avec le temps de polissage (chute de $20 \mu \mathrm{m}$ en $15 \mathrm{~min}$ ), mais les échantillons de dureté plus élevée possèdent une développée plus faible à l'instant initial $(5 \mu \mathrm{m})$. Par contre, la décroissance de la développée est plus importante pour les échantillons de plus faible dureté. Il semblerait que nous atteignons une asymptote dont la valeur dépend de la dureté du matériau (507 $\mu \mathrm{m}$ pour l'échantillon A, $502 \mu \mathrm{m}$ pour les échantillons de dureté inférieure à 200). La rugosité des échantillons grenaillés $(t=0)$ est plus faible en amplitude pour les échantillons à faible dureté (différence de $0,4 \mu \mathrm{m}$ pour le $R_{\mathrm{a}}, 0,7 \mu \mathrm{m}$ pour le $R_{\mathrm{pm}}$ et de $0,5 \mu \mathrm{m}$ pour le $R_{\mathrm{i}}$ ), mais présente plus de pics (différence supérieure à 300 pics). L'opération de grenaillage modifie la rugosité différemment en fonction de la dureté de l'échantillon. Par contre, dès les premiers instants de polissage par le procédé Ecoclean, les paramètres $R_{\mathrm{a}}$ et $R_{\mathrm{pm}}$ ne sont plus corrélés avec la dureté alors que nous notons une 

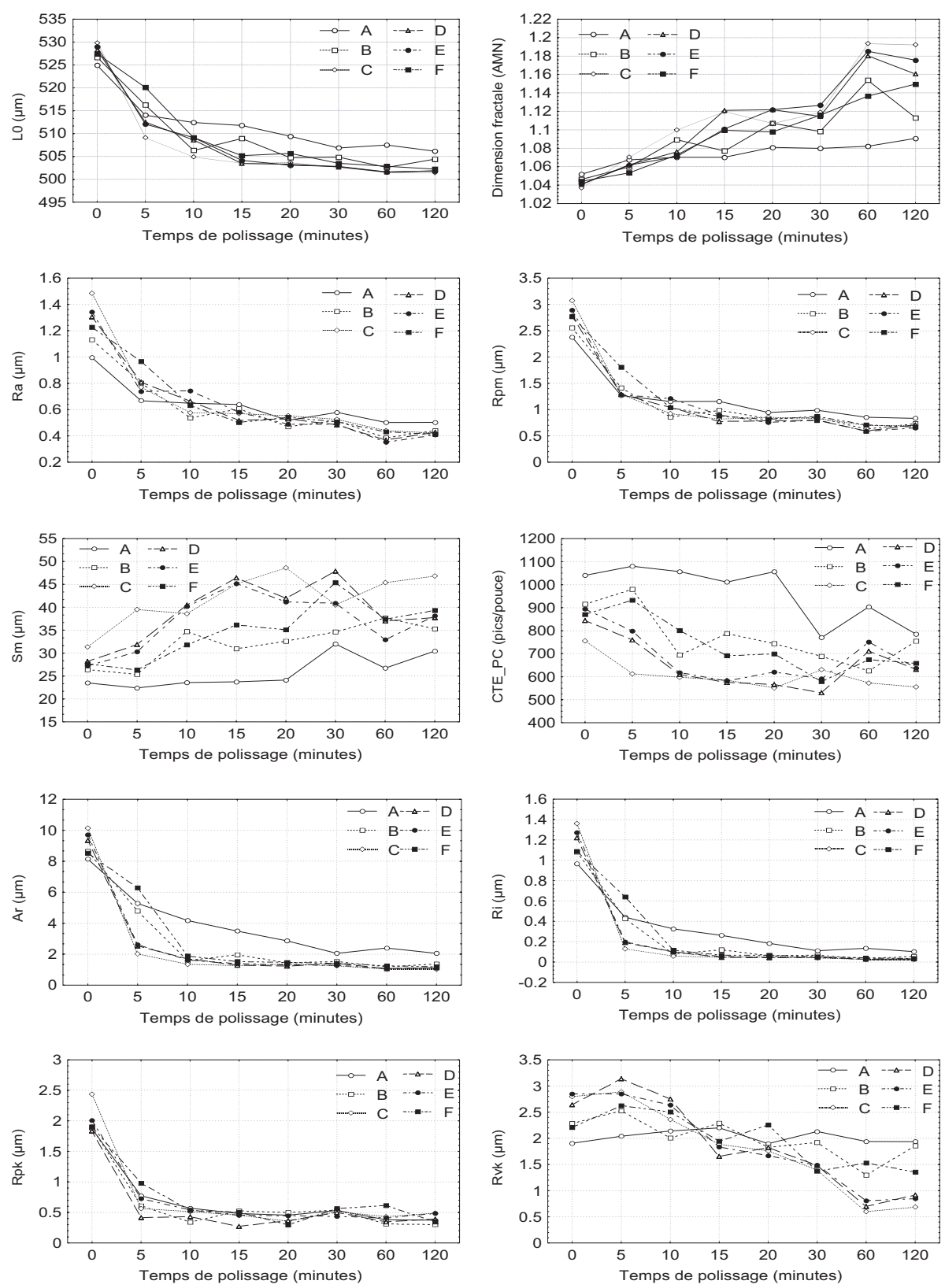

Fig. 5. Étude des paramètres de rugosité de la campagne 1. Chaque point est la moyenne calculée à partir de 10 mesures (10 mesures par essai) pour chaque matériau.

différence significative pour le paramètre d'amplitude $R_{\mathrm{i}}(0,1 \mu \mathrm{m})$. Comme le $R_{\mathrm{i}}$ est l'amplitude de la rugosité obtenue par la méthode des profils par la décomposition Cnomo : la dureté de l'échantillon n'influence pas l'amplitude globale de la rugosité du profil, mais plutôt une amplitude locale. Par contre, le paramètre d'amplitude qui discrimine au mieux l'influence de la dureté sur le polissage Ecoclean est le $R_{\mathrm{vk}}$ (différence de $1 \mu \mathrm{m}$ ). En effet, une rugosité de vallées est plus difficile à imprégner qu'une rugosité de pics et particulièrement pour des duretés élevées. En effet, les vallées de l'échantillon A $(H V=380)$ ne sont pas endommagées $\left(R_{\mathrm{vk}} \approx 2 \mu \mathrm{m}\right)$, l'échantillon $\mathrm{B}$ $(H V=280)$ voit ses vallées légèrement érodées (chute de $R_{\mathrm{vk}}$ de $\left.0,5 \mu \mathrm{m}\right)$, alors que les autres échantillons
$(H V<200)$ voient nettement le paramètre $R_{\mathrm{pk}}$ chuter (chute de $2 \mu \mathrm{m}$ ). Le $R_{\mathrm{pk}}$ atteint la valeur asymptotique de $0,5 \mu \mathrm{m}$ après un temps de polissage de $5^{\prime}$ pour les échantillons les moins durs $(\mathrm{C}, \mathrm{D}, \mathrm{E}, \mathrm{F})$ et cette même valeur asymptotique après un temps de polissage de $10^{\prime}$ pour les échantillons plus durs (A et B).

Le paramètre $A_{\mathrm{r}}$ montre que l'espace entre les pics (des motifs) chute de $9 \mu \mathrm{m}$ à $2 \mu \mathrm{m}$ et peut être imputé à une érosion localisée des pics car le $S_{\mathrm{m}}$ augmente, caractérisant la formation de pics plus massifs. De plus, il est plus difficile de créer des pics massifs par sablage pour les échantillons durs (A et B), caractérisés par des paramètres $A_{\mathrm{r}}$ et $R_{\mathrm{i}}$ plus faibles. Par contre, une analyse plus fine des paramètres de rugosité montre que le graphe 
de la dimension fractale calculée par la méthode AMN, en fonction du temps de polissage, possède une allure qui diffère complètement de celle rencontrée avec les autres paramètres. En effet, la dimension fractale croît quasi linéairement avec le temps de polissage. Ce paramètre, mathématiquement indépendant de l'amplitude du profil, caractérise, dans cette étude, l'apparition d'une microrugosité assez chaotique, mais ayant une amplitude si faible que les autres paramètres ne peuvent la quantifier. En effet, la méthode AMN analyse l'ordre de la rugosité à l'aide d'une fenêtre glissante de largeur inférieure à $2 \mu \mathrm{m}$ et ce paramètre est faiblement influencé numériquement par les variations d'amplitude plus globales. La dimension fractale croît moins rapidement avec le temps de polissage si le matériau est plus dur : la micro-rugosité serait plus délicate à imprimer sur un matériau de plus faible dureté. L'apparition d'une micro-rugosité semble être confirmée par une remontée du nombre de pics après un temps d'érosion de 30 min. Dans un premier temps, les pics plus massifs sont érodés, masquant alors la mesure de cette micro-rugosité. Puis une fois ceux-ci érodés, le procédé algorithmique de comptage des pics détecte les pics de cette micro-rugosité (créée progressivement au cours du temps). Nous voyons apparaître un graphe en U caractéristique de la combinaison de 2 phénomènes concurrentiels : érosion des pics massifs et création de pics localisés.

Remarque : L'échantillon E de dureté la moins élevée $(H V=160)$, est plus résistant à l'endommagement que les échantillons $\mathrm{C}$ et $\mathrm{D}$, de dureté plus élevée $(H V=190)$.

\subsection{Campagne 2}

Dans cette campagne, l'acide est remplacé par de l'eau. Nous constatons un classement des paramètres influents calculé par l'analyse de la variance bootstrapée semblable à celui obtenu pour les deux campagnes antérieures. De même, la valeur maximale du $F$ est très élevée (1000) représentant une modification très prononcée de la surface. En traçant l'évolution des 10 paramètres retenus en fonction du temps de polissage, nous constatons les faits suivants (Fig. 6) :

1) Il n'y a pas de différence pour les paramètres d'amplitude, que l'érosion ait lieu en milieu acide ou aqueux. Les asymptotes, après $120 \mathrm{~h}$ de polissage, ont des valeurs communes quels que soient les paramètres. Nous avons particulièrement analysé les deux matériaux moins nobles (A et $\mathrm{E}$ ) qui devraient être plus sensibles au phénomène de corrosion et pour lesquels l'absence de milieu acide devrait se faire ressentir plus fortement. Pourtant, une analyse statistique confirme le fait qu'il n'y a pas d'influence du liquide de polissage.

2) La croissance linéaire de la dimension fractale avec le temps de polissage est confirmée ainsi que l'allure en U de la courbe du nombre de pics. Nous avions émis l'hypothèse que ces deux tendances étaient dues à l'apparition d'une micro-rugosité. Cette dernière ne peut donc être reliée à la corrosion électrochimique de la surface.
Il semblerait donc que la présence d'acide soit totalement inefficace pour cette gamme de matériaux.

\subsection{Campagne 3}

Dans cet essai, seule l'influence de l'acide sur une modification de la morphologie de surface est étudiée. Par une analyse de variance bootstrapée (Fig. 7), plus de la moitié des paramètres de rugosité ne discriminent pas l'effet corrosif de la solution. Certes, quelques paramètres discriminent les surfaces, mais ont une valeur de $F$ très faible $(F \approx 10)$, alors que les campagnes précédentes montraient des valeurs de $F$ supérieures à 100. Cependant, le paramètre $\mathrm{G}$, caractérisant le nombre de points d'inflexion du profil divisé par le nombre de points du profil est nettement plus discriminant que les autres. Ce paramètre, calculé par une méthode numérique développée au sein de notre laboratoire, discriminait le phénomène d'usure par le procédé de polissage Ecoclean lors des trois campagnes précédentes mais était loin d'être un paramètre très discriminant.

En traçant (Fig. 8) l'évolution du nombre du points d'inflexion en fonction du temps de maintien de l'échantillon dans la solution acide, nous constatons une chute de ce nombre de $9,8 \%(t=0)$ à $9,4 \%$ $(t=4 \mathrm{~h})$. Les points d'inflexion sont calculés d'après 4 points consécutifs du profil qui représentent une largeur de $0,12 \mu \mathrm{m}$ et donc un phénomène très localisé. Il faut noter que ce paramètre est invariant avec l'amplitude mais est très sensible au pas d'échantillonnage. Nous pouvons donc émettre deux hypothèses :

1) Une légère corrosion lisse le profil sur moins d'un dixième de micron. En effet, les angles très vifs sont érodés par un effet de pointes connu en électrochimie. Ce phénomène peut être amplifié par écrouissage au cours de l'écrouissage de la surface. Cependant, ce phénomène n'est pas détecté par le calcul de la dimension fractale qui n'est pas considéré comme statistiquement discriminant.

2) Suite au grenaillage, certains débris de surface, faiblement liés au substrat, sont « décrochés » de la surface par le mouvement turbulent du fluide et diminuent donc le nombre de points d'inflexion du profil.

Nous penchons pour la deuxième interprétation car l'analyse statistique ne détecte aucune différence du paramètre $\mathrm{G}$ entre les différentes classes de matériaux qui n'ont pas le même potentiel de corrosion. Comme la présence d'acide n'affecte pas la rugosité globale, nous considérons que l'acide n'influence pas la rugosité de surface des échantillons (Fig. 9).

\subsection{Analyse du polissage manuel}

Nous allons comparer les états de surface du polissage manuel avec ceux obtenus par ce nouveau procédé de polissage. 

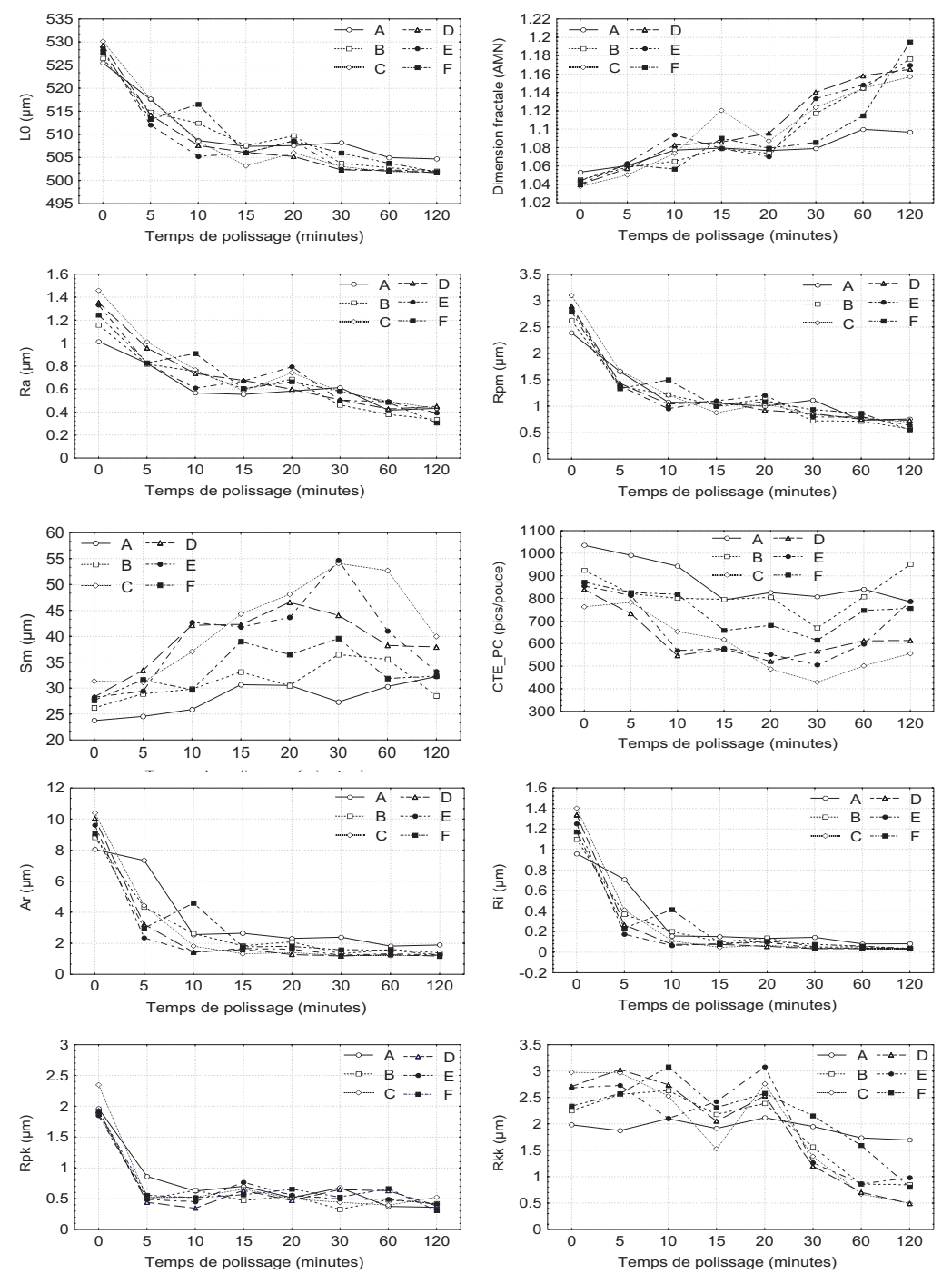

Fig. 6. Étude des paramètres de rugosité de la campagne 2. Chaque point est la moyenne calculée à partir de $2 \times 10$ mesures (10 mesures par essai) pour chaque matériau.

\subsubsection{Caractérisation de l'aspect hétérogène du polissage manuel}

Nous constatons que le polissage manuel donne à l'échantillon un aspect de brillance hétérogène que n'ont pas les échantillons polis par la machine Ecoclean. Comment alors quantifier cette hétérogénéité de brillance? Nous avons montré que la dimension fractale AMN de la surface d'un matériau caractérise parfaitement sa brillance [5]. Cependant, l'échantillon est de taille très faible et il est délicat expérimentalement de mesurer les zones de brillance différentes. Si nous admettons que la dimension fractale caractérise la brillance, alors un échantillon de brillance hétérogène présentera des dimensions fractales hétérogènes. Par conséquent, si nous effectuons un nombre de mesures de profils assez important, nous pouvons espérer quantifier cette hétérogénéité par une augmentation de la dispersion de la dimension fractale. Pour quantifier cette dispersion, l'écart type des 10 mesures de la dimension fractale est calculé pour chaque échantillon poli manuellement et l'écart type des 10 mesures pour les échantillons polis par le procédé après un temps de $20 \mathrm{~min}$ (les dimensions fractales des surfaces entre polissage manuel et polissage automatique sont égales pour ce temps de polissage). Nous constatons que l'écart type de la dimension fractale des échantillons polis manuellement est près du double de celui obtenu avec les échantillons polis avec le procédé Ecoclean. La variation de la dimension fractale calculée par la méthode AMN caractérise alors les aspects de facettes de l'échantillon poli manuellement (Tab. 3).

\subsubsection{Comparaison des deux méthodes de polissage : manuelle et Ecoclean}

La comparaison des deux structures de rugosité (polissage manuel et automatique) n'est pas triviale. En effet, la morphologie des surfaces est différente et il est donc délicat de comparer ces rugosités avec un seul critère. 


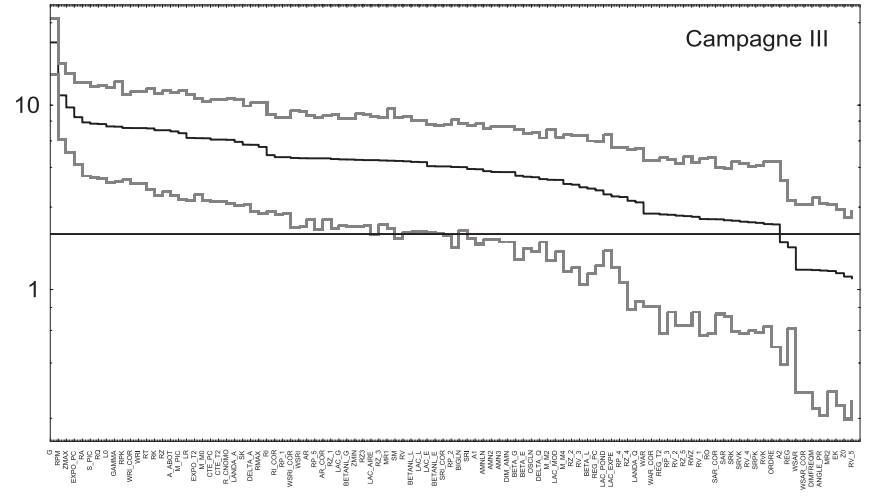

Fig. 7. Classement des paramètres de rugosité de la campagne 3 par l'analyse de la variance bootstrapée. Les paramètres sont classés par $\boldsymbol{F}$ décroissant (plus influent vers le moins influent). Les limites des intervalles de confiance à $95 \%$ sont représentées en lignes discontinues. La ligne horizontale représente la valeur critique du $\boldsymbol{F}$ en dessous de laquelle le paramètre de rugosité n'est plus significatif.

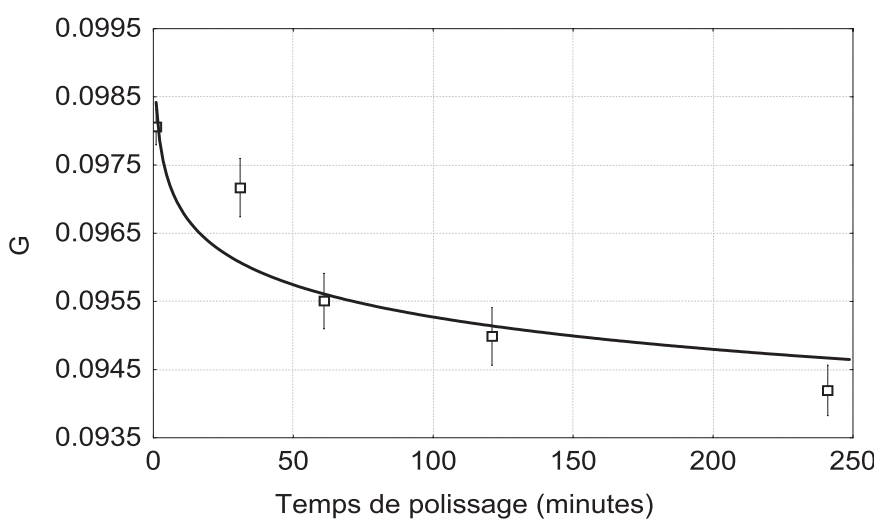

Fig. 8. Analyse de l'évolution du ratio du nombre de points d'inflexion du profil de rugosité de la campagne 3. Chaque point est la moyenne calculée à partir de $10 \times 6$ mesures (10 mesures par essai, 6 matériaux) tous matériaux confondus.

Pour analyser ces deux textures nous allons procéder de la manière suivante :

1) Comme il n'existe pas de différence hautement significative entre les essais des deux premières campagnes, elles sont regroupées en une seule qui donne une population de taille plus importante qui permettra d'affiner les statistiques.

2) Les 10 critères de rugosité qui caractérisent la surface sont calculés pour le polissage manuel ainsi que les moyennes par gamme d'échantillons.

3) L'évolution de ces moyennes est tracée en fonction du temps de polissage pour chaque matériau et y est ajoutée la moyenne des mesures faites en polissage manuel (notée MA sur la Fig. 10).

4) Le test de Student permet d'analyser les égalités des moyennes entre les paramètres de rugosité des surfaces polies manuellement et les surfaces polies par le procédé Ecoclean et ce, pour chaque temps de polissage.

Dans le tableau 4, nous avons regroupé les valeurs des moyennes des paramètres « polissage manuel »
Tableau 3. Statistiques descriptives (moyennes et écarttypes) de la dimension fractale (méthode AMN) calculée sur des surfaces polies manuellement et par le procédé Ecoclean (temps de polissage de $20 \mathrm{~min}$ ).

\begin{tabular}{ccccc}
\hline & \multicolumn{2}{c}{ Écart-type } & \multicolumn{2}{c}{ Moyenne } \\
& Manuelle & Ecoclean & Manuelle & Ecoclean \\
\hline $\mathrm{B}$ & 0,028 & 0,011 & 1,131 & 1,097 \\
$\mathrm{C}$ & 0,031 & 0,016 & 1,101 & 1,101 \\
$\mathrm{~A}$ & 0,054 & 0,025 & 1,088 & 1,100 \\
$\mathrm{D}$ & 0,037 & 0,022 & 1,103 & 1,108 \\
$\mathrm{E}$ & 0,036 & 0,026 & 1,096 & 1,119 \\
$\mathrm{~F}$ & 0,018 & 0,013 & 1,074 & 1,077 \\
Moyenne & 0,034 & 0,019 & 1,099 & 1,100 \\
\hline
\end{tabular}

$\left(2^{\mathrm{e}}\right.$ colonne) et les moyennes «polissage Ecoclean » (colonnes de 3 à 12). Sous chaque moyenne des paramètres, nous inscrivons la probabilité critique du $t$ de Student (en italique dans le tableau). Physiquement, ce chiffre représente la probabilité d'erreur d'affirmer que les moyennes sont différentes alors qu'elles sont égales. Ce chiffre sera d'autant plus élevé que la moyenne entre le paramètre caractérisant la surface polie mécaniquement sera proche du même paramètre caractérisant la surface polie par le procédé Ecoclean. Nous considérons le non-rejet de l'égalité des moyennes dès que cette probabilité sera supérieure à 0,05 (en gras dans le tableau). Par exemple, la surface polie manuellement possède le même $S_{\mathrm{m}}$ que la surface polie avec le procédé Ecoclean pendant une durée de 5 min. La surface grenaillée $(t=0)$ possède un $S_{\mathrm{m}}$ plus faible $(27,57 \mu \mathrm{m}$ contre $30,36 \mu \mathrm{m})$, mais après $10 \mathrm{~min}$ de polissage Ecoclean, le $S_{\mathrm{m}}$ de la surface est plus élevé. Le tableau est ordonné par égalité des paramètres croissants avec le temps de polissage.

Une visualisation rapide du tableau permet d'affirmer qu'il n'est pas possible d'obtenir par le procédé Ecoclean une surface équivalente à la surface polie manuellement. En effet, si les surfaces étaient identiques, alors elles auraient statistiquement les mêmes paramètres de rugosité et donc il existerait au moins une colonne dans laquelle tous les $t$ de Student seraient supérieurs à 0,05 (qui aurait, par conséquent donné le temps de polissage nécessaire pour obtenir une surface équivalente). Or il n'existe pas de temps de polissage qui permette d'obtenir cette égalité statistique des paramètres, et les surfaces sont donc de natures différentes. Par contre, il est toujours possible, par un choix adéquat du temps de polissage du procédé Ecoclean, d'obtenir une surface ayant le même paramètre de rugosité que le procédé de polissage manuel.

Le polissage manuel écrête les « pics de surface » introduits lors du grenaillage $\left(R_{\mathrm{pk}_{\text {grenaillé }}}: 1,981 \mu \mathrm{m}, R_{\mathrm{pk}_{\text {Manuel }}}\right.$ : $0,475 \mu \mathrm{m})$ et il est nécessaire de polir plus de $10 \mathrm{~min}$ avec le procédé Ecoclean pour atteindre cet écrêtage. Le polissage manuel crée une rugosité à pics assez élevée. En effet, leur nombre est identique à celui de l'échantillon grenaillé (881 pics/inch) qui est confirmé par une distance entre les pics plus courte $\left(S_{\mathrm{m}_{\text {Manuel }}}: 30,36 \mu \mathrm{m}\right)$. Pour quantifier l'aspect assez « aigu » de la rugosité, nous analysons la dimension fractale des profils et nous constatons que celle-ci 

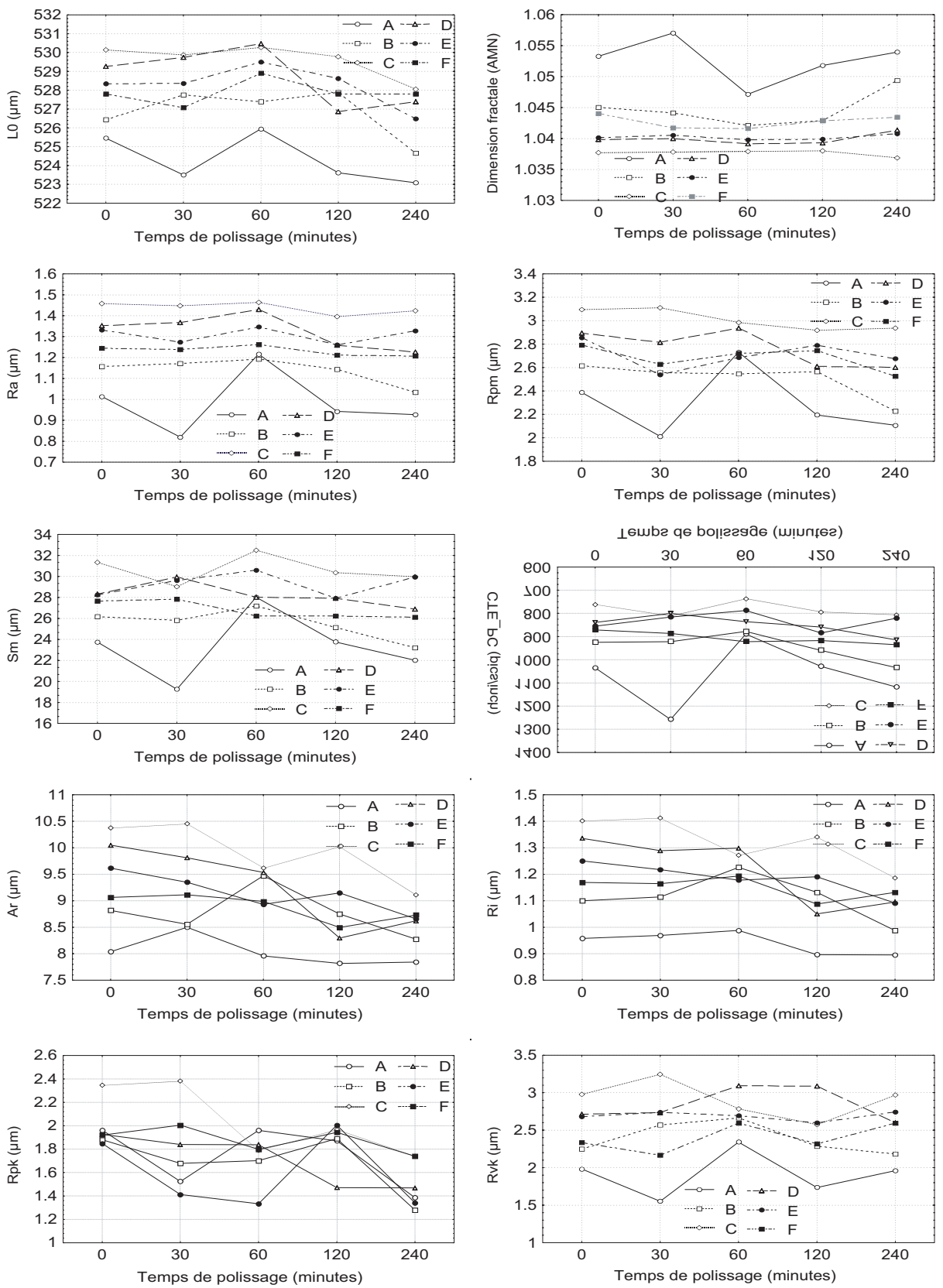

Fig. 9. Étude des paramètres de rugosité de la campagne 3. Chaque point est la moyenne calculée à partir de 10 mesures (10 mesures par essai) pour chaque matériau.

est plus élevée que pour le profil initial $\left(\Delta_{\text {Sablé }}: 1,04\right.$, $\left.\Delta_{\text {Manuel }}: 1,10\right)$. Par contre, la surface développée est plus faible après polissage manuel $\left(L_{0_{\text {Sablé }}}: 527 \mu \mathrm{m}\right.$, $\left.L_{0_{\text {Manuel }}}: 505 \mu \mathrm{m}\right)$ et s'explique par la chute importante de l'amplitude de la rugosité $\left(R_{\mathrm{pm}_{\text {grenaillé }}}: 2,77 \mu \mathrm{m}\right.$, $\left.R_{\text {pm }_{\text {Manuel }}}: 0,84 \mu \mathrm{m}\right)$. Cette chute assez brutale est principalement due à la destruction de pics massifs, formés par sablage dès les premiers instants du polissage manuel $\left(A_{\mathrm{r}_{\text {grenaille }}}: 9,3 \mu \mathrm{m}, A_{\mathrm{r}_{\text {Manuel }}}: 2,2 \mu \mathrm{m}\right)$. Mais le polissage manuel ne peut donner une structure aussi « lisse » que le procédé Ecoclean. En effet, après 30 min de polissage Ecoclean, tous les paramètres d'amplitude sont inférieurs ou égaux à ceux obtenus par polissage manuel mais leur nombre de pics est très nettement inférieur

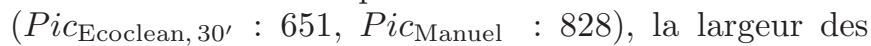
pics étant nettement plus étendue $\left(S_{\mathrm{m}_{\text {Ecoclean }}, 30^{\prime}}: 39,5\right.$, $\left.S_{\mathrm{m}_{\text {Manuel }}}: 30,4\right)$.

Nous allons rechercher le paramètre de rugosité qui discrimine au mieux les deux procédés de polissage. Pour mener cette analyse, nous procédons à un regroupement en deux classes :

Classe 1 : Échantillons polis manuellement,

Classe 2 : Échantillons polis Ecoclean, tous temps confondus.

Nous procédons à l'analyse de la variance bootstrapée pour trouver le paramètre le plus discriminant. 

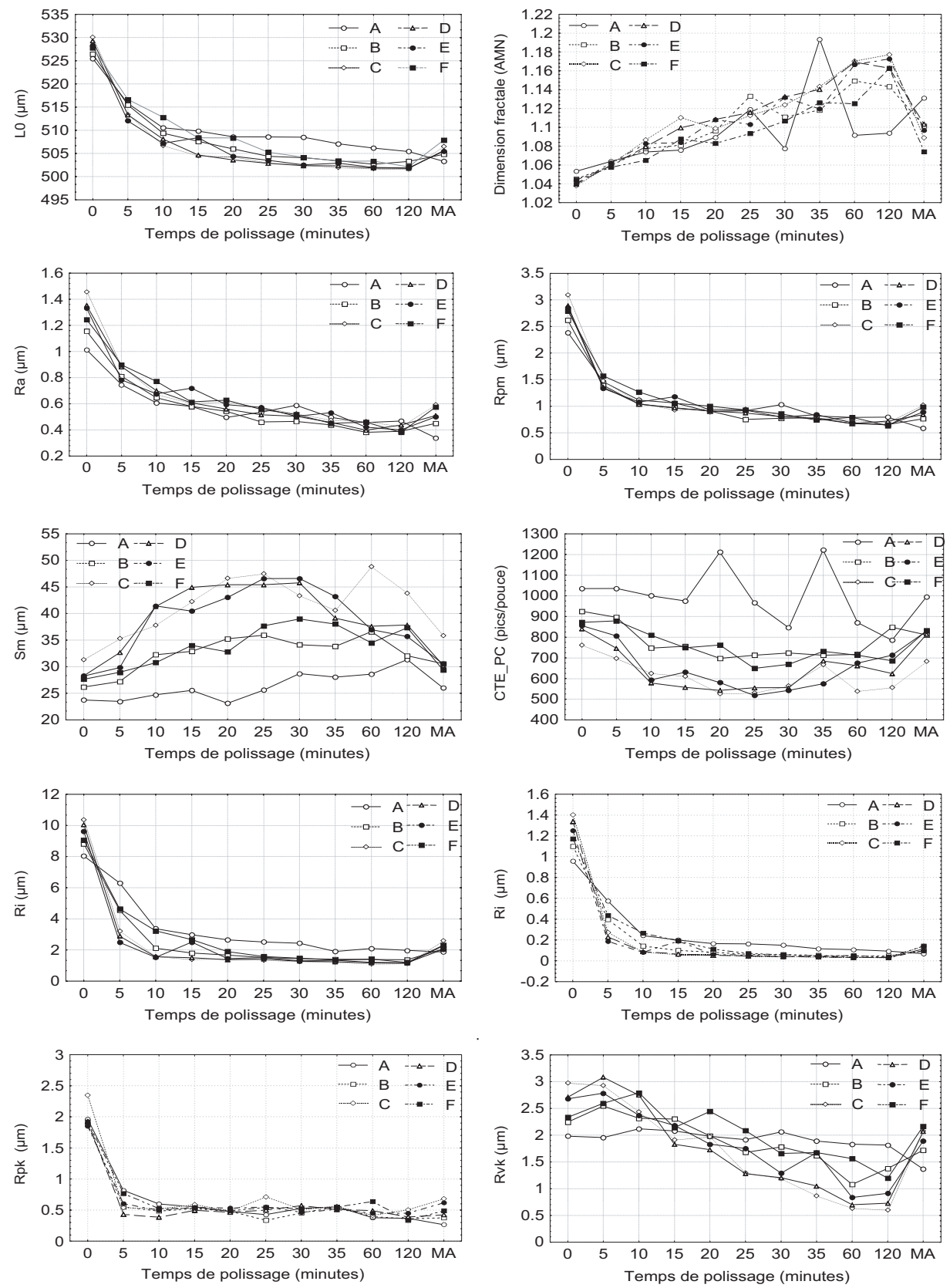

Fig. 10. Étude des paramètres de rugosité des campagnes 1, 2 et 3. Chaque point est la moyenne calculée à partir de 40 mesures (4 séries d'essais, 10 mesures par essai) pour chaque matériau. L'échantillon noté MA correspond au polissage manuel.

Le paramètre $S_{\mathrm{k}}$ est le plus discriminant. En effet, pour les profils grenaillés, le $S_{\mathrm{k}}$ est nul $\left(S_{\mathrm{k}_{\text {grenaillé }}}:-0,04\right)$. Or, un $S_{\mathrm{k}}$ nul est caractéristique d'une distribution des amplitudes gaussiennes, habituelle à une structure sablée ou grenaillée. Un $S_{\mathrm{k}}$ négatif traduit une dissymétrie de la courbe des amplitudes caractéristique d'une suppression assez importante des pics. Or, le $S_{\mathrm{k}}$ d'une surface polie manuellement est nettement inférieur au $S_{\mathrm{k}}$ des surfaces polies par le procédé Ecoclean (Tab. 5), et ce, quel que soit le temps de polissage $\left(S_{\mathrm{k}_{\text {Ecoclean }}}:-0,50, S_{\mathrm{k}_{\text {Manuel }}}:-0,91\right)$. Pour visualiser la courbe des amplitudes, nous allons tracer l'histogramme de tous les profils pour des temps de polissage différents et des nuances différentes. Pour cela, tous les profils sont redressés par une droite des moindres carrés, puis la répartition des points redressés permet de tracer la fonction de densité empirique. Pour chaque histogramme nous calculons la moyenne (qui est toujours nulle) et l'écart type qui nous permettent de tracer la courbe de Gauss associée à chaque histogramme. En visualisant les histogrammes nous constatons que :

1) Il n'y a pas de points aberrants dans ces histogrammes et donc toutes les mesures expérimentales ont été effectuées correctement.

2) Tous les échantillons grenaillés ont une densité gaussienne. 
Tableau 4. Analyse de la matrice de comparaison des moyennes entre polissage manuel et polissage machine (campagnes 1,2 et 3). Le chiffre en gras signifie le non rejet de l'égalité des paramètres au seuil de confiance de $95 \%$.

\begin{tabular}{|c|c|c|c|c|c|c|c|c|c|c|c|}
\hline \multirow{2}{*}{\multicolumn{2}{|c|}{$\begin{array}{c}\text { Moyenne } \\
\text { Manuel }\end{array}$}} & \multicolumn{10}{|c|}{ Temps de polissage Ecoclean (minutes) } \\
\hline & & 0 & 5 & 10 & 15 & 20 & 25 & 30 & 35 & 60 & 120 \\
\hline \multirow[t]{2}{*}{ Pics } & 828 & 881 & 842 & 725 & 714 & 709 & 655 & 651 & 771 & 698 & 710 \\
\hline & & 0,0542 & 0,6547 & 0,0010 & 0,0000 & 0,0000 & 0,0000 & 0,0000 & 0,0739 & 0,0000 & 0,0003 \\
\hline \multirow[t]{2}{*}{$S_{\mathrm{m}}$} & 30,36 & 27,57 & 29,59 & 34,72 & 36,63 & 38,02 & 39,78 & 39,50 & 36,93 & 36,97 & 35,98 \\
\hline & & 0,0177 & 0,5664 & 0,0012 & 0,0000 & 0,0000 & 0,0000 & 0,0000 & 0,0000 & 0,0000 & 0,0001 \\
\hline \multirow[t]{2}{*}{$R_{\mathrm{pk}}$} & 0,475 & 1,981 & 0,617 & 0,502 & 0,541 & 0,488 & 0,512 & 0,509 & 0,538 & 0,461 & 0,401 \\
\hline & & 0,0000 & 0,0036 & 0,5896 & 0,1236 & 0,7577 & 0,4497 & 0,4266 & 0,2042 & 0,7659 & 0,1399 \\
\hline \multirow[t]{2}{*}{$A_{\mathrm{r}}$} & 2,1800 & 9,322 & 3,995 & 2,228 & 2,146 & 1,726 & 1,644 & 1,536 & 1,413 & 1,400 & 1,351 \\
\hline & & 0,0000 & 0,0000 & 0,7200 & 0,6663 & 0,0000 & 0,0000 & 0,0000 & 0,0000 & 0,0000 & 0,0000 \\
\hline \multirow[t]{2}{*}{$R_{\mathrm{i}}$} & 0,109 & 1,201 & 0,346 & 0,149 & 0,134 & 0,086 & 0,073 & 0,064 & 0,056 & 0,049 & 0,046 \\
\hline & & 0,0000 & 0,0000 & 0,0082 & 0,0625 & 0,0803 & 0,0176 & 0,0007 & 0,0005 & 0,0001 & 0,0001 \\
\hline \multirow[t]{2}{*}{$R_{\mathrm{vk}}$} & 1,881 & 2,486 & 2,651 & 2,464 & 2,076 & $\mathbf{1 , 9 9 0}$ & 1,668 & 1,534 & 1,470 & 1,114 & 1,132 \\
\hline & & 0,0000 & 0,0000 & 0,0000 & 0,0076 & 0,1367 & 0,0108 & 0,0000 & 0,0000 & 0,0000 & 0,0000 \\
\hline \multirow[t]{2}{*}{$\mathrm{A}_{\mathrm{mn}}$} & 1,099 & 1,043 & 1,060 & 1,077 & 1,089 & 1,097 & 1,112 & 1,113 & 1,140 & 1,144 & 1,150 \\
\hline & & 0,0000 & 0,0000 & 0,0000 & 0,0044 & 0,5814 & 0,0006 & 0,0000 & 0,0000 & 0,0000 & 0,0000 \\
\hline \multirow[t]{2}{*}{$L_{0}$} & 505,6 & 527,8 & 514,4 & 509,1 & 507,2 & 505,8 & 504,5 & 504,0 & 503,5 & 502,9 & 502,8 \\
\hline & & 0,0000 & 0,0000 & 0,0000 & 0,0000 & 0,4506 & 0,0008 & 0,0000 & 0,0000 & 0,0000 & 0,0000 \\
\hline \multirow[t]{2}{*}{$R_{p m}$} & 0,846 & 2,772 & 1,431 & 1,101 & 1,028 & 0,935 & 0,891 & 0,851 & 0,788 & 0,725 & 0,694 \\
\hline & & 0,0000 & 0,0000 & 0,0000 & 0,0000 & 0,0001 & 0,0845 & 0,8166 & 0,0268 & 0,0000 & 0,0000 \\
\hline \multirow[t]{2}{*}{$R_{\mathrm{a}}$} & 0,494 & 1,258 & 0,834 & 0,678 & 0,617 & 0,574 & 0,535 & 0,517 & 0,474 & 0,429 & 0,418 \\
\hline & & 0,0000 & 0,0000 & 0,0000 & 0,0000 & 0,0000 & 0,0049 & 0,0698 & 0,1779 & 0,0000 & 0,0000 \\
\hline
\end{tabular}

Tableau 5. Analyse de la matrice de comparaison des moyennes pour le paramètre $S m$ entre polissage manuel et polissage machine (campagnes 1, 2 et 3 ).

\begin{tabular}{cccccccccccc}
\hline & \multicolumn{10}{c}{ Temps de polissage Ecoclean (minutes) } \\
\hline & Manuel & 0 & 5 & 10 & 15 & 20 & 25 & 30 & 35 & 60 & 120 \\
\hline$S_{\mathrm{k}}$ & $-0,919$ & $-0,046$ & $-0,689$ &,- 818 &,- 681 &,- 684 &,- 570 &,- 469 &,- 441 &,- 355 &,- 418 \\
\hline & 0,0000 & 0,0000 & 0,0000 & 0,0413 & 0,0000 & 0,0000 & 0,0000 & 0,0000 & 0,0000 & 0,0000 & 0,0000 \\
\hline
\end{tabular}

3) Dès les cinq premières min de polissage, l'histogramme devient dissymétrique et cette dissymétrie est d'autant plus marquée que le matériau utilisé possède une faible dureté. Cette dissymétrie, par l'introduction d'un skewness négatif, traduit l'érosion des pics.

4) À l'exception de l'échantillon A, les histogrammes des amplitudes tendent à se rapprocher de la distribution gaussienne pour des temps de polissage supérieurs à une heure.

5) À amplitude de rugosité égale (à l'exception de l'échantillon A), l'échantillon poli manuellement possède une dissymétrie nettement plus prononcée que l'échantillon poli à l'aide du procédé Ecoclean.

\section{Analyse des surfaces en microscopie électronique à balayage (MEB)}

\subsection{Acquisition des images MEB}

Nous avons choisi d'étudier l'échantillon Cerapall-2 (B) pour 5 temps d'érosion de la campagne 1. Puis ces échantillons sont analysés au MEB. Les images sont numérisées sous format TIF $(1024 \times 1024,256$ niveau de gris) pour être ensuite analysées par le programme d'analyse fractale d'image développé au sein de notre laboratoire. La figure 11 représente les images obtenues pour des temps de polissage de $0,5,15,30$ et $60 \mathrm{~min}$. En observant visuellement les échantillons, l'effet du polissage semble lisser la surface. Il apparaît des cavités nettement prononcées après un temps de polissage de $15^{\prime}$, puis ces cavités diminuent de taille au cours du polissage. Pour caractériser ces cavités, de nouvelles acquisitions sont effectuées pour les isoler en faisant varier les grossissements de 100 à 20000 . Une cinquantaine de cavités sont ainsi numérisées pour chaque temps de polissage, ces images sont alors seuillées manuellement (seuillage par limite supérieure sur les niveaux de gris).

\subsection{Analyse de la répartition des aires des cavités}

La figure 12 représente la mesure des aires des cavités pour les différents temps de polissage Ecoclean, une analyse statistique élémentaire montre que ces aires présentent une population homogène, de répartition lognormale confirmée par les tests statistiques de Kolmogorov. Les mesures sont homogènes et le diagramme des moyennes auxquelles sont associés les intervalles de confiance montre que :

1) Les aires mesurées sont faibles à l'instant initial, $1120 \mu \mathrm{m}^{2} \pm 150$. 


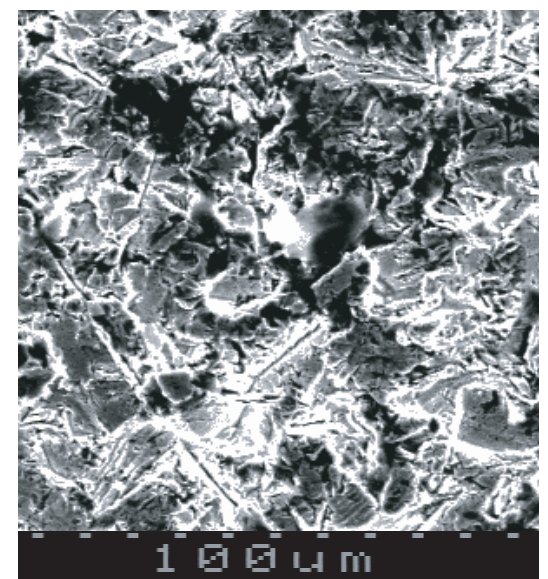

(a)

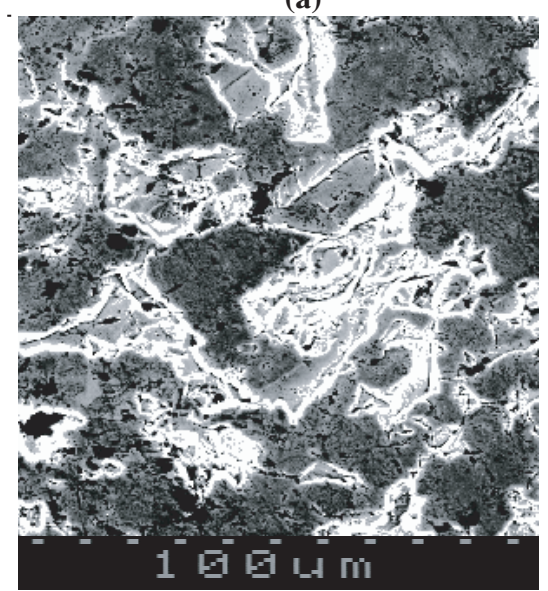

(c)

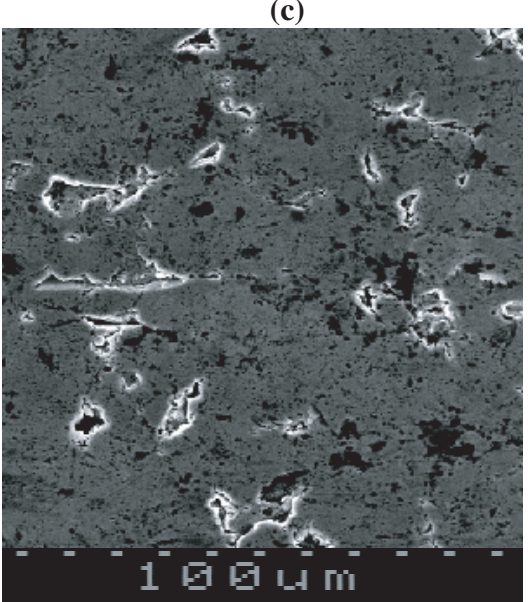

(e)

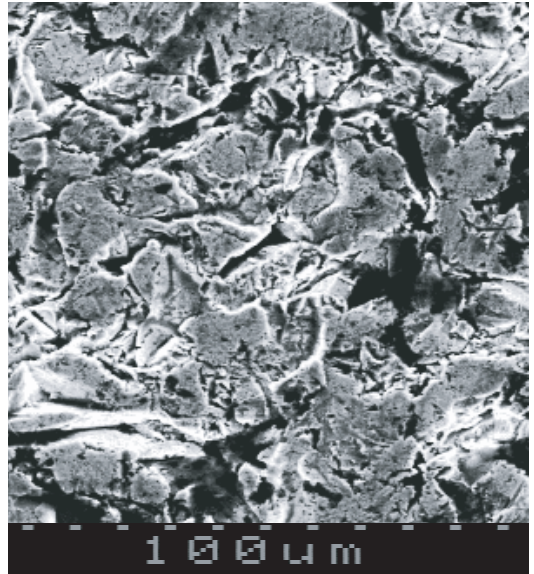

(a)

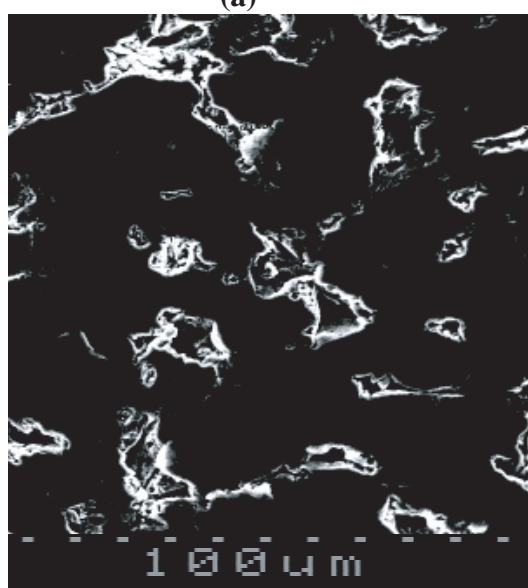

(d)

Fig. 11. Images MEB en 256 niveaux de gris obtenus après différents temps de polissage pour l'échantillon Cerapall 2 (B). (a) : grenaillé $\left(t=0^{\prime}\right) /$ Shot blasted; (b) $: t=5^{\prime} ;(\mathrm{c}): t=15^{\prime} ;(\mathrm{d}): t=30^{\prime} ;(\mathrm{e}): t=60^{\prime}$.

2) Elles atteignent au maximum $2180 \mu \mathrm{m}^{2} \pm 150$ après un temps de polissage inférieur ou égal à 5 min.

3) Après ce maximum, les aires diminuent pour atteindre une asymptote d'environ $1260 \mu \mathrm{m}^{2} \pm 150$.

Le tableau des Tests de Student confirme qu'il n'est plus possible de déceler une modification de l'aire des cavités après un polissage supérieur à 30 min.

\subsection{Analyse de la courbe d'Abbott des profils}

Nous allons analyser la rugosité des échantillons Cerapall 2 pour expliquer les résultats observés au MEB. La figure 1 représente les profils de rugosité de l'usure du Cerepall 2 subissant le polissage sous solution acide pour la même gamme de temps que celle étudiée au MEB. Les courbes d'Abbott sont utilisées pour analyser l'amplitude 


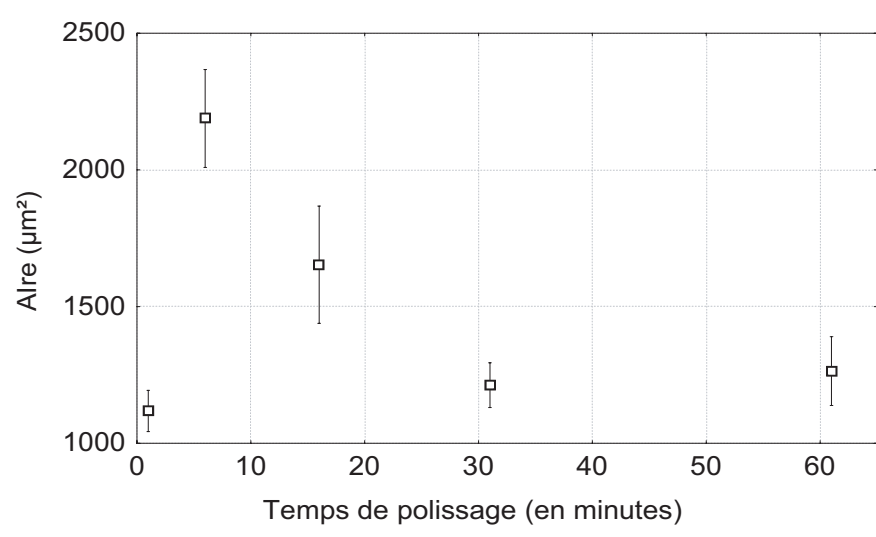

\begin{tabular}{|cc|c|c|c|c|c|}
\hline & & $\{1\}$ & $\{2\}$ & $\{3\}$ & $\{4\}$ & $\{5\}$ \\
\hline \multicolumn{2}{|c|}{ Moyenne } & 1118 & 2188 & 1653 & 1212 & 1264 \\
\hline 0 & $\{1\}$ & & $5,7008 \mathrm{E}-08$ & 0,00962086 & 0,6158476 & 0,48724911 \\
\hline 5 & $\{2\}$ & $5,7008 \mathrm{E}-08$ & & 0,00844775 & $1,7996 \mathrm{E}-07$ & $9,6127 \mathrm{E}-06$ \\
\hline 15 & $\{3\}$ & 0,00962086 & 0,00844775 & & 0,02546487 & 0,07452144 \\
\hline 30 & $\{4\}$ & 0,6158476 & $1,7996 \mathrm{E}-07$ & 0,02546487 & & $\mathbf{0 , 7 9 7 5 8 8 2 2}$ \\
\hline 60 & $\{5\}$ & 0,48724911 & $9,6127 \mathrm{E}-06$ & 0,07452144 & $\mathbf{0 , 7 9 7 5 8 8 2 2}$ & \\
\hline
\end{tabular}

Fig. 12. Relation entre l'aire des cavités mesurée sur le MEB sous différents grossissements X1000 à X20000, après seuillage manuel en tout ou rien, et le temps de polissage pour l'échantillon Cerapall 2 (B). L'aire mesurée correspond à l'aire du polygône des pixels yardstickés développée dans notre programme de calcul de dimension fractale par la méthode des îles. Dans le tableau ci-dessus, le chiffre en gras signifie le non rejet de l'égalité des aires mesurées au seuil de confiance de $95 \%$.

de la rugosité, en utilisant le logiciel de calcul des paramètres décrit en section 3 . Cependant, pour pouvoir visualiser toutes les courbes d'Abbott sur une seule courbe, nous avons ajouté une composante à notre système informatique de traitement. Pour une gamme de temps de polissage, les courbes d'Abbott sont calculées. Pour chaque courbe d'Abbott, nous disposons de 250 couples de valeurs numériques $\%$ de matière - Amplitude des pics $(A H D(c), c)$. De part le mode de tracé de la courbe d'Abbott, il est très peu probable d'obtenir les mêmes valeurs de $A H D(c)$ ou $c$. Les valeurs de $A H D(c)$ sont regroupées en 101 classes de même largeur. $A H D(c)$ qui peuvent prendre les valeurs 0\%, $1 \%, \ldots, 99 \%, 100 \%$. Pour chaque classe de pourcentage de matière, la moyenne des interceptés est calculée permettant le tracé d'une courbe d'Abbott moyenne des profils (Fig. 13). L'avantage de cette méthode est de disposer d'une courbe d'Abbott beaucoup plus stable car moyennée sur un grand nombre de profils et donc les conclusions obtenues visuellement de la courbe sont robustes. En analysant l'évolution des paramètres d'Abbott sur le processus d'érosion en fonction du temps de polissage (Fig. 14), il est possible de proposer un classement de ces paramètres par ordre d'influence :

$R_{\mathrm{k}}>$ Ordonnée $=$ Pente $>R_{\mathrm{pk}}>R_{\mathrm{vk}}=A_{2}=A_{1}>$ $M_{\mathrm{r}}>M_{\mathrm{r} 1}$

Puisque $M_{\mathrm{r} 1}$ est constant quel que soit le temps d'érosion $(8,9 \%)$, la fin du régime linéaire de la rugosité reste inchangée. Pour $M_{\mathrm{r} 2}$, nous constatons que l'évolution est inversement liée à l'aire des cavités

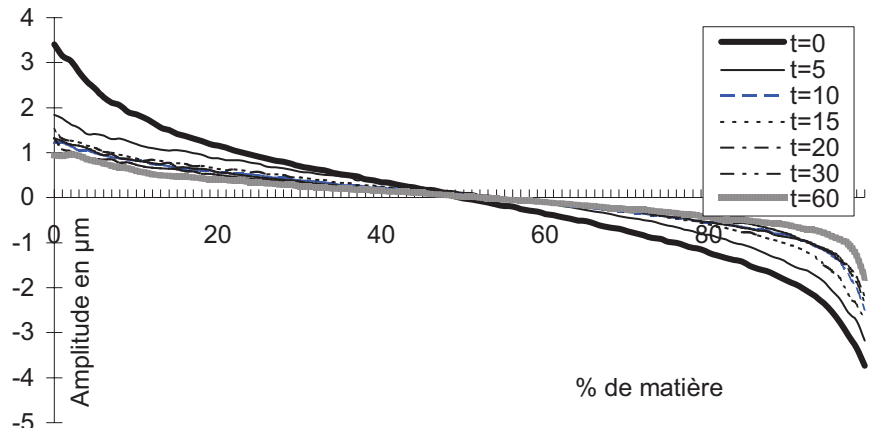

Fig. 13. Évolution de la courbe moyenne d'Abbott des profils de rugosité de l'échantillon Cerapall 2 au cours du processus de polissage. Les profils sont effectués après un temps de polissage de $0^{\prime}, 5^{\prime}, 10^{\prime}, 15^{\prime}, 20^{\prime}, 30^{\prime}$ et $60^{\prime}$.

(Fig. 12) : quand l'aire des cavités croît, $M_{r 2}$ décroît. En effet, l'érosion des cavités augmente la zone linéaire de la région centrale de la rugosité en diminuant le régime des vallées. Ce résultat est confirmé par l'évolution du coefficient $A_{2}$, qui représente un «volume » de vallées décroissant au cours du temps. Le processus érode les vallées comme le confirme la chute de 1,2 $\mu \mathrm{m}$ du coefficient $R_{\mathrm{vk}}$ (de $2,54 \mu \mathrm{m}$ à $\left.1,37 \mu \mathrm{m}\right)$. L'analyse de la matrice de Student, montre, après un polissage de $20 \mathrm{~min}$, qu'il n'y a plus érosion des vallées comme le confirme l'analyse des aires des images. Nous notons une chute très rapide du $R_{\mathrm{pk}}$ dès les premiers instants du polissage. Statistiquement (utilisation des matrices des tests de Student), le $R_{\text {pk }}$ est constant après un temps d'érosion maximum de 5 min ce qui montre que le processus d'usure détruit les parties «saillantes » des pics de rugosité très rapidement; après érosion de ces pics, la morphologie supérieure de la rugosité reste inchangée. Par contre, la rugosité centrale $\left(R_{\mathrm{k}}\right)$ chute progressivement de $2,5 \mu \mathrm{m}$ pour atteindre une asymptote à $1,2 \mu \mathrm{m}$. L'analyse de la matrice des comparaisons du $t$ de Student montre que le $R_{\mathrm{k}}$ n'évolue plus après $25 \mathrm{~min}$.

\section{Discussion}

La figure 15 permet de constater que le polissage diminue l'amplitude de la rugosité d'une manière significative. En effet, la surface grenaillée possède un $R_{\mathrm{a}}$ de $1,3 \mu \mathrm{m}$ qui chute après 5 min à $0,65 \mu \mathrm{m}$. Un polissage prolongé ne permettrait pas de diminuer significativement l'amplitude de la rugosité. L'interprétation de ce phénomène peut être liée à la taille des aiguilles, trop importante pour pouvoir éroder plus efficacement la surface. De plus, ce phénomène doit être influencé par la perte de charge en surface : après un laps de temps assez court, la vitesse $\mathrm{du}$ liquide est proportionnelle à la vitesse des aiguilles au loin de la pièce à polir. À la surface de l'échantillon de dimension $L \times L$, il existe une couche limite d'épaisseur $\delta$ avec $\delta=4,92 \sqrt{\frac{L \mu}{v \rho}} \approx 0,025 \sqrt{L}$ qui est voisine, dans cette expérience, de $2 \mathrm{~mm}$. Comme les aspérités sont dans cette couche limite (partie rotationnelle visqueuse 

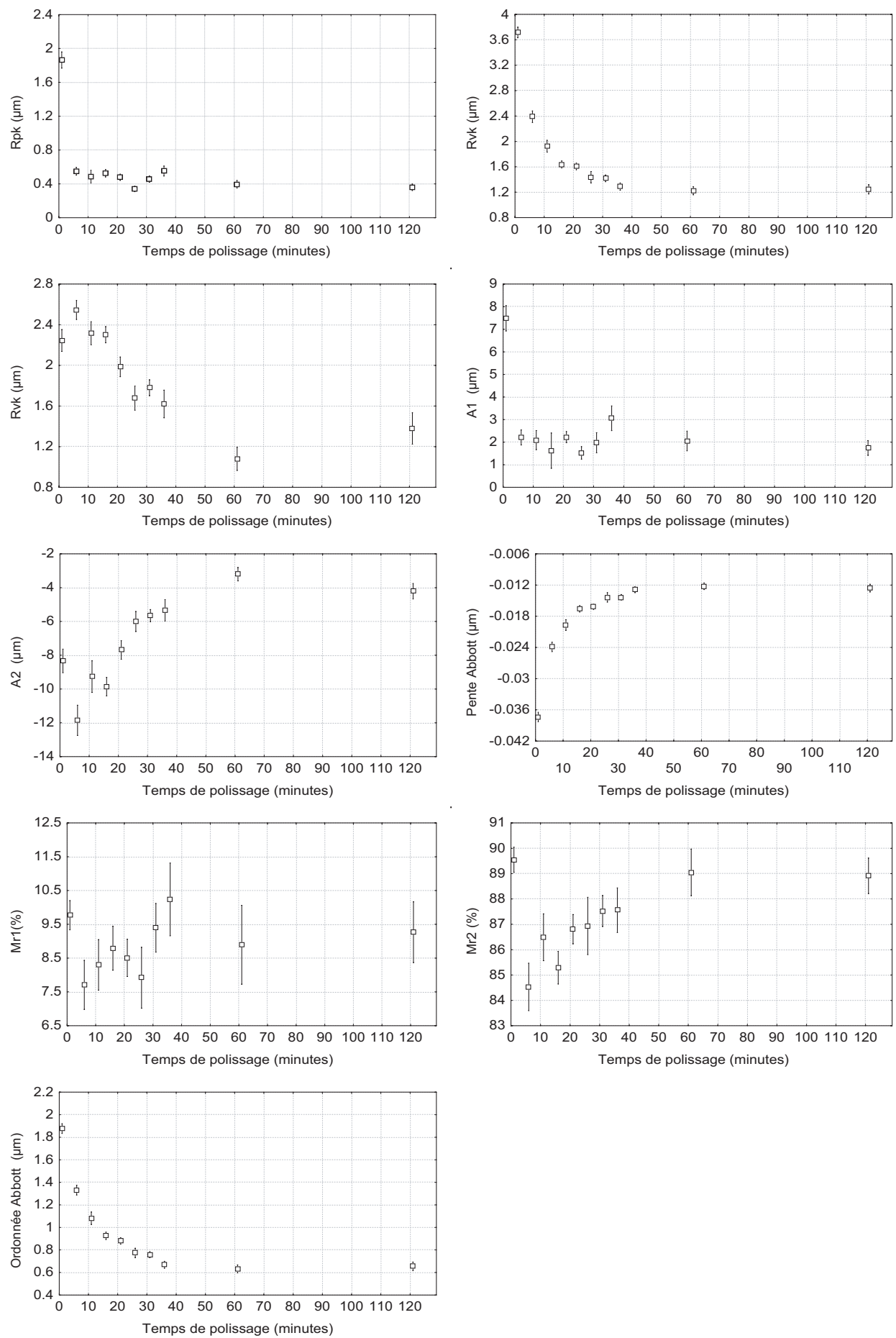

Fig. 14. Évolution des paramètres moyens liés aux courbes d'Abbott des profils de rugosité de l'échantillon Cerapall 2 au cours du processus de polissage.

de l'écoulement), le fluide possède une vitesse influencée par la rugosité. Les aiguilles ont tendance à frapper l'échantillon d'autant plus fréquemment que les pics sont élevés et nombreux, ce qui entraîne la décroissance non linéaire de l'amplitude de la rugosité.

Les pics initiaux assez importants sont rapidement érodés puisque nous enregistrons une perte de 200 pics par pouce. Il s'agit d'une érosion des pics les moins massifs due aux chocs des aiguilles. La chute est assez franche et la courbe atteint un minimum au bout de $25 \mathrm{~min}$ (650 pics.pouce ${ }^{-2}$ ). Passé ce délai, le nombre de pics recommence à augmenter. La présence d'une courbe en forme de parabole est souvent représentative de deux phénomènes concurrentiels qui prédominent chacun dans un intervalle de temps donné. Le premier phénomène, responsable de la chute du nombre de pics, est sans conteste 

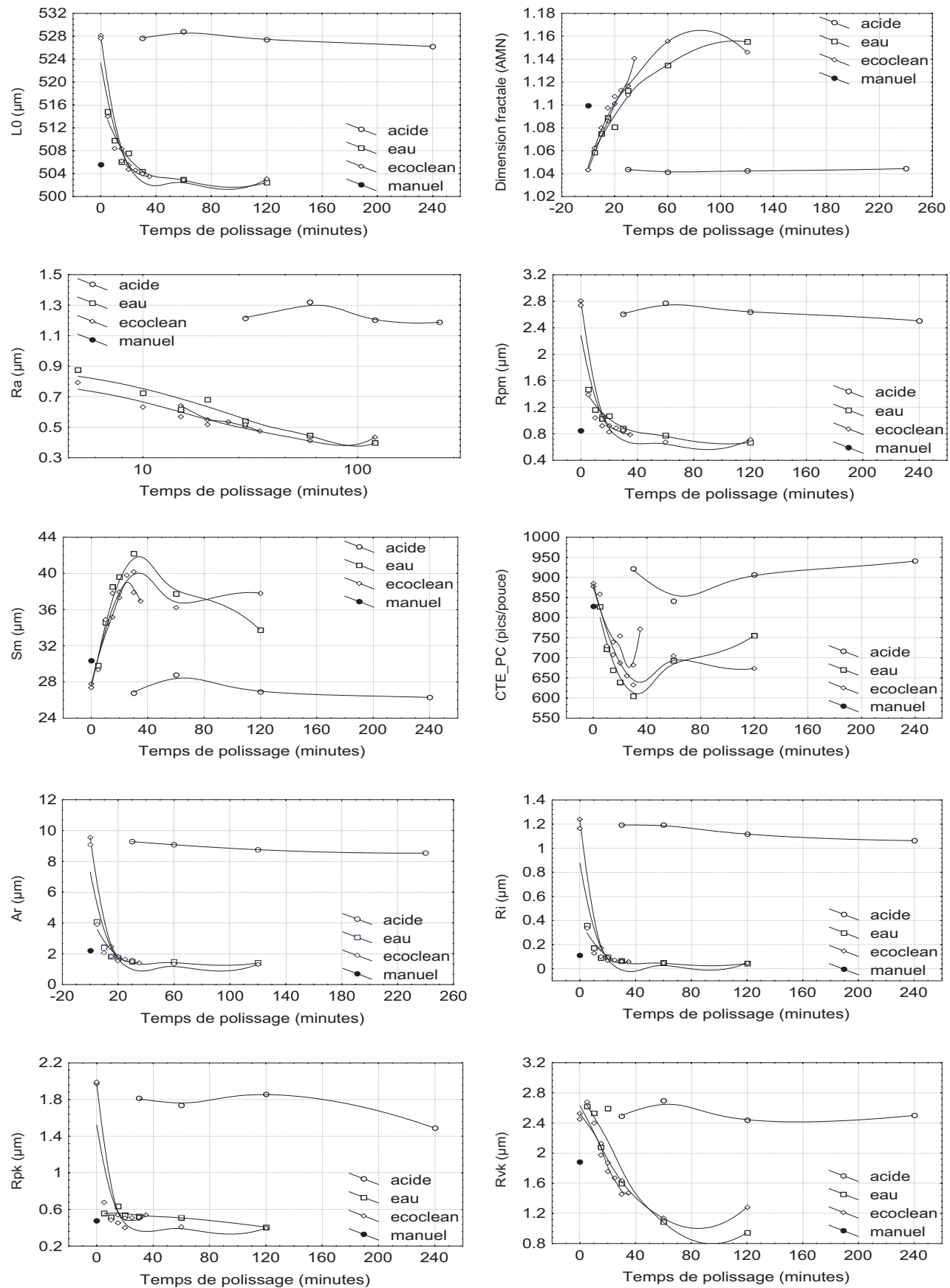

Fig. 15. Moyenne des paramètres de rugosité obtenue pour tous les essais tous matériaux confondus.

l'érosion due au choc des aiguilles sur l'échantillon. Le deuxième phénomène est plus délicat à expliquer. En effet, il faut constater que le $R_{\mathrm{a}}$ ne présente pas de minimum et décroît avant ou après $25 \mathrm{~min}$ avec la même tendance logarithmique. Ce deuxième phénomène, qui ne crée pas de rugosité supplémentaire, est concentré à la micro-rugosité. Cette micro-rugosité est parfaitement caractérisée par la dimension fractale calculée par la méthode AMN qui augmente avec le temps de polissage Ecoclean.

L'acide n'a aucun rôle sur l'érosion des pics massifs ni sur l'apparition de la micro-rugosité. Même couplé à un endommagement mécanique, le milieu acide ne joue aucun rôle. Ce phénomène est dû à la composition chimique des matériaux qui sont tous à base d'éléments nobles et donc très faiblement oxydables. Par conséquent, l'augmentation de la dimension fractale n'est pas due au phénomène de corrosion de surface qui présente une structure fractale de part sa nature diffusive [2]. Par contre, bien qu'il faille confirmer ce résultat, il est possible que la présence de l'acide lisse la surface sur une échelle très faible que nous estimons inférieure à $0,1 \mu \mathrm{m}$, mais ce phénomène n'est pas quantifiable sur l'amplitude de la rugosité elle-même ni sur le nombre de pics et de vallées. Comme l'acide n'est pas influent, nous déconseillons son utilisation susceptible 
de polluer la surface, pollution nuisible à une application ultérieure dans le cadre du génie biomédical.

Nous pensons que l'origine de la micro-rugosité est liée à l'arrachement de la matière lors du choc des aiguilles. En effet, nous avons montré que l'amplitude de la rugosité, initialement gaussienne due au grenaillage, devient dissymétrique et est imputable à la suppression de pics assez massifs. Mais celle-ci redevient gaussienne après prolongation du polissage. Donc, le fait d'obtenir une amplitude gaussienne quand l'amplitude de la rugosité n'évolue plus, montre que la surface est formée par une succession de mécanismes qui peut être imputée à de l'arrachement de matière. Cet enlèvement de matière par le procédé de polissage Ecoclean peut être schématisé par une découpe du profil en une succession de plans de coupe parallèles au substrat. En effet, l'analyse fractale sur des images MEB des cavités [1] présentes sur les échantillons, montre qu'elles sont les résidus des vallées initiales de l'échantillon (avant polissage). Par contre, l'érosion de ces vallées ne sera jamais totale et sera bornée à une aire d'environ $1200 \mu \mathrm{m}^{2}$ et la profondeur d'environ $1 \mu \mathrm{m}$. Un modèle de simulation numérique à caractère stochastique confirme cette hypothèse.

L'analyse de profil tridimensionnel montre que les pics deviennent moins aigus au cours du temps de polissage. Par contre, la chute de l'amplitude de la rugosité est liée à la dureté initiale du matériau. En effet, plus le matériau est dur, moins rapide sera l'érosion par ce procédé bien qu'en fin de polissage, nous atteignions une amplitude de rugosité commune quelle que soit la dureté du matériau (ceci n'est pas le cas lors du polissage manuel). Cependant, il paraît délicat de tenter un polissage par ce procédé pour des matériaux de dureté supérieure à $300 \mathrm{HV}$ au vu du comportement du matériau A $(380 H V)$. Celui-ci est nettement moins sensible à ce type de polissage que les autres échantillons. Il est donc nécessaire d'ajuster le temps de polissage en fonction de la dureté du matériau. La durée de 30 min de polissage pour les alliages dentaires semble offrir un excellent compromis entre la chute optimale de l'amplitude et la faible amplitude de la microrugosité.

Ce procédé de polissage est très reproductible. En effet nous n'avons rencontré pratiquement aucune différence entre les différents échantillons que ce soit au niveau des amplitudes, du nombre de pics ou de l'aspect fractal du profil. D'ailleurs, les images MEB confirment un endommagement uniforme sur toute la surface. Nous expliquons ce phénomène par le très grand nombre de chocs pendant lesquels les aiguilles créent l'érosion et par la conservation assez constante de l'énergie cinétique lors du choc (couche turbulente du fluide et vitesse de rotation de la machine constante). Le polissage par abrasion n'exerce pas un endommagement constant car ce dernier, responsable du polissage, est nettement plus influencé par la topographie de la surface. En effet, la pression exercée sur un instrument de polissage traditionnel engendre une contrainte à la surface du matériau qui dépend de la rugosité. Le polissage manuel offre une surface qui présente une structure de brillance hétérogène que nous avons caractérisée par une variabilité de la mesure de la dimension fractale mesurée par AMN. Par contre, nous constatons que le procédé Ecoclean polit les surfaces uniformément. De plus, le polissage manuel présente une répartition des amplitudes non-gaussiennes qui peut nuire pour des applications futures. Le polissage manuel présente, à amplitude de rugosité égale, une structure plus chaotique caractérisée par la présence de pics plus nombreux, moins érodés et d'angles plus vifs. Cette structure désordonnée s'explique par la succession de stries de polissage qui peut nuire à certaines utilisations (adhésion). Nous observons également que la rugosité du polissage manuel est anisotrope car due à la présence de stries de polissage qui peuvent donner des sillons d'une profondeur de $5 \mu \mathrm{m}$ pour les échantillons à dureté élevée (présence d'un bourrelet). Les résultats du polissage manuel dépendent de la dureté du matériau. Plus la dureté de l'échantillon est faible, plus la rugosité est profonde et l'amplitude de la rugosité peut varier du simple au double. Par contre, l'amplitude de la rugosité obtenue par polissage Ecoclean ne dépend pas du matériau si l'érosion est suffisamment prolongée et si la dureté de l'échantillon n'est pas excessive $(<300 H V)$. Le fait que la rugosité d'une surface polie dépende de la dureté est compréhensible si nous admettons que l'enlèvement de matière a lieu après un processus d'écrouissage. De ce fait, les stries formées vont créer un bourrelet dont la hauteur dépend de la dureté (plus la dureté est élevée, plus le bourrelet est important). Ce bourrelet sera ensuite érodé, mais donnera lieu à d'autres stries et ainsi de suite ... Par contre, pour le procédé Ecoclean, la vitesse des chocs des aiguilles sur le matériau est très élevée $\left(\approx 4 \mathrm{~m} . \mathrm{s}^{-1}\right)$ et très localisée avec un angle d'attaque assez faible sur le plan de référence de la surface. L'enlèvement de matière a lieu avec la création d'une faible zone plastique. Cette hypothèse serait à confirmer par des mesures de contraintes résiduelles pour tester si la profondeur de la zone écrouie est plus faible par ce procédé de polissage. Une analyse EDX devrait également être menée sur la surface de l'échantillon pour quantifier les éventuelles pollutions introduites par les aiguilles de polissage.

\section{Conclusion}

Dans cette étude, nous avons étudié un nouveau procédé de polissage qui offre certains avantages par rapport aux procédés classiques (homogénéité du polissage, facilité d'emploi, résultats répétitifs). Pour cette étude, un très grand nombre d'essais a dû être effectué pour stabiliser les statistiques obtenues. Les paramètres de rugosité disponibles pour caractériser l'état de surface obtenu sont nombreux, certains peuvent permettre d'expliquer des mécanismes d'obtention de surface et doivent être sélectionnés par des critères statistiques (analyse de variance, régression ...). De même, les processus physiques qui sont à l'origine de la création (ou modification) ne peuvent être expliqués uniquement à l'aide des paramètres d'amplitude de la rugosité et il est souvent nécessaire d'associer un ou plusieurs paramètres. 
L'analyse de la variance bootstrapée nous a permis de rechercher les paramètres influents parmi la multitude de paramètres disponibles; il s'agit donc d'un outil statistique très utile pour limiter à quelques paramètres pertinents la topographie d'une surface. De même, l'étude des surfaces au MEB fournit une vision complémentaire sur la topographie de la surface. L'emploi du calcul de la dimension fractale par la méthode AMN a permis de quantifier trois phénomènes distincts : hétérogénéité de la brillance d'un échantillon, quantification de l'influence de la dureté du matériau sur le procédé de grenaillage et détection de la présence d'une micro-rugosité de surface.

Finalement, cette étude semble montrer que la polisseuse Ecoclean est un excellent tribomètre (bien que ce ne fût pas sa fonctionnalité première) qui peut permettre d'aborder les phénomènes de tribologie en milieu liquide, avec un mécanisme d'endommagement original, susceptible de nuancer certaines conclusions obtenues avec des mesures issues d'un tribomètre conventionnel.

\section{Références}

[1] M. Bigerelle, K. Anselme, A. Iost, Analyse fractale des surfaces. Applications au polissage d'alliages précieux et à la prolifération cellulaire, Innov. Techn. Biol. Med. 20 (1999) 319-330

[2] M. Bigerelle, Thèse de Doctorat, ENSAM Lille (1999), et Habilitation à Diriger les Recherches, UST Lille 1 (2003)

[3] M. Bigerelle, D. Najjar, A. Iost, The computer-based bootstrap method as a tool to select a relevant surface, Wear 254 (2003) 450-460

[4] M. Bigerelle, A. Iost, Calcul de la dimension fractale d'un profil par la méthode des autocorrélations moyennées normées (AMN), C.R. Académie des Sciences Paris série IIB 323 (1996) 669-675

[5] M. Bigerelle, D. Najjar, A. Iost, Relevance of roughness parameters for description and modelling of machined surfaces, J. Material Science 38 (2003) 2525-2536

Retrouvez nos articles sur le site : www.edpsciences.org/meca 